\title{
Global Solutions of the Relativistic Euler Equations
}

\author{
Joel Smoller ${ }^{1}$, Blake Temple ${ }^{2}$ \\ ${ }^{1}$ Department of Mathematics, University of Michigan, Ann Arbor, MI 48109, USA; Supported \\ in part by NSF Applied Mathematics Grant Number DMS-89-05205 \\ 2 Department of Mathematics, University of California, Davis, Davis CA 95616, USA; Supported \\ in part by NSF Applied Mathematics Grant Number DMS-86-13450
}

Received April 24, 1992

\begin{abstract}
We demonstrate the existence of solutions with shocks for the equations describing a perfect fluid in special relativity, namely, $\operatorname{div} T=0$, where $T^{i j}=\left(p+\rho c^{2}\right) u^{i} u^{j}+p \eta^{i j}$ is the stress energy tensor for the fluid. Here, $p$ denotes the pressure, $u$ the 4-velocity, $\rho$ the mass-energy density of the fluid, $\eta^{i j}$ the flat Minkowski metric, and $c$ the speed of light. We assume that the equation of state is given by $p=\sigma^{2} \rho$, where $\sigma^{2}$, the sound speed, is constant. For these equations, we construct bounded weak solutions of the initial value problem in two dimensional Minkowski spacetime, for any initial data of finite total variation. The analysis is based on showing that the total variation of the variable $\ln (\rho)$ is non-increasing on approximate weak solutions generated by Glimm's method, and so this quantity, unique to equations of this type, plays a role similar to an energy function. We also show that the weak solutions $\left(\rho\left(x^{0}, x^{1}\right), v\left(x^{0}, x^{1}\right)\right)$ themselves satisfy the Lorentz invariant estimates $\operatorname{Var}\left\{\ln \left(\rho\left(x^{0}, \cdot\right)\right\}<V_{0}\right.$ and $\operatorname{Var}\left\{\ln \frac{c+v\left(x^{0}, \cdot\right)}{c-v\left(x^{0}, \cdot\right)}\right\}<V_{1}$ for all $x^{0} \geqq 0$, where $V_{0}$ and $V_{1}$ are Lorentz invariant constants that depend only on the total variation of the initial data, and $v$ is the classical velocity. The equation of state $p=\left(c^{2} / 3\right) \rho$ describes a gas of highly relativistic particles in several important general relativistic models which describe the evolution of stars.
\end{abstract}

\section{Introduction}

We consider the relativistic equations for a perfect fluid in Minkowski spacetime,

$$
\operatorname{div} T=0,
$$

where

$$
T^{i j}=\left(p+\rho c^{2}\right) u^{i} u^{j}+p \eta^{i j}
$$

denotes the stress-energy tensor for the fluid. Recall that in Minkowski spacetime,

$$
\operatorname{div} T \equiv T_{j, i}^{i},
$$


where we use the Einstein summation convention and assume summation over repeated up-down indices. The notation ", $i$ " denotes differentiation with respect to the variable $x^{i}$, and in general all indices run from 0 to 3 with $x^{0} \equiv c t$. In (2), $c$ denotes the speed of light, $p$ the pressure, $u$ the 4-velocity of the fluid particle (the velocity of the frame of isotropy of the perfect fluid), $\rho$ the mass-energy density of the fluid (as measured in units of mass in a reference frame moving with the fluid particle), and $\eta^{i j} \equiv \eta_{i j} \equiv \operatorname{diag}(-1,1,1,1)$ denotes the flat Minkowski metric. In the case of a barotropic gas, $p$ is given by an explicit function of $\rho$, and this defines the equation of state. Barotropic fluids are important in the study of stellar evolution in general relativity. ${ }^{3}$ In the case of barotropic flow, system (1) describes a system of four conservation laws in the four unknowns $\rho$ and $u$. Recall that since $u=$ $(1 / c) d x / d \tau$, ( $\tau$ is the proper time, $u$ is a unit four vector in Minkowski space) it follows that $\left(u^{0}\right)^{2}-\sum_{1}^{3}\left(u^{\alpha}\right)^{2}=1$, and thus only three of the quantities $u^{0}, \ldots, u^{3}$ are independent.

As a special example of barotropic flow, the equation of state $p=\left(c^{2} / 3\right) \rho$ arises in several important relativistic settings. In particular, the equation of state $p=$ $\left(c^{2} / 3\right) \rho$ follows directly from the Stefan-Boltzmann law when a gas is in thermodynamical equilibrium with radiation and the radiation energy density greatly exceeds the total gas energy density. Indeed, the significance of the special case $p=\left(c^{2} / 3\right) \rho$ is further discussed in the following quote from the recent book of A.M. Anile [1], starting at the bottom of p. 12:

An example of astrophysical interest ... is provided by a gas in local thermodynamical equilibrium with radiation when the radiation energy density greatly exceeds the total gas energy density. Since for radiation, one has [the Stephan-Boltzmann law] $\rho=C_{B} T^{4}$ and $p=(1 / 3) C_{B} T^{4}$ [where $c=1, T=$ temperature and $C_{B}$ denotes the Stephan-Boltzmann constant], when the radiation energy density dominates, the fluid obeys the equation of state $p=(1 / 3) \rho$. . . Other examples are provided by a fluid of massless neutrinos ... or a fluid of ultra-relativistic electron-positron pairs .... In both cases, the pressure is given by $p=(1 / 3) \rho$. It is interesting to notice that in the early universe, at sufficiently high temperatures, all the particles become relativistic. Therefore, the equation of state $p=(1 / 3) \rho$ would [also] be applicable under these circumstances.

The equation of state $p=\left(c^{2} / 3\right) \rho$ has also been important in the study of gravitational collapse because it can be derived as a model for the equation of state in a dense Neutron star. This derivation is given in Weinberg [20], p. 320. This

${ }^{3}$ We quote Weinberg [20], p. 301 (Weinberg uses the term "isentropic" in place of "barotropic"):

[the assumption that the] star is isentropic is valid for two very different kinds of stars: (A) Stars at Absolute Zero. When a star exhausts its thermonuclear fuel it can become a white dwarf, or a neutron star, in which the temperature is essentially at absolute zero. According to Nernst's theorem, the entropy per nucleon will then be zero throughout the star. (B) Stars in Convective Equilibrium. If the most efficient mechanism for energy transfer within the star is convection, then in equilibrium the entropy per nucleon must be nearly constant throughout the star, because otherwise a small element of fluid ... could gain or lose energy when transported from one part of the star to another, and convection would therefore disturb the energy distribution. The supermassive stars are generally presumed to be in convective equilibrium... The importance of these assumptions lies in the fact that the pressure $p$. . may be regarded as a function of the energy density $\rho$ alone 
result is also discussed in the famous works of Oppenheimer and Snyder and Oppenheimer and Volkoff $[14,15]$ on gravitational collapse; see also $[6,11,18,19]$.

The equation of state $p=\sigma^{2} \rho$ also describes the equation of state in isothermal flow. The isothermal case is valid in the early stages of stellar formation. If one imagines a slowly collapsing cloud of interstellar gas or dust particles, the collapse reaches a stage where the mean free path for photon transmission within the cloud becomes small enough so that the scattering of photons is a significant effect. During the period where the motion within the cloud remains relatively small, the photon scattering has the effect of equalizing the temperature throughout the cloud, and thus $p=\sigma^{2} \rho$ is valid. ${ }^{4}$

Observe also that a general equation of state $p=p(\rho)$ can be approximated by one of form (6) by linearizing the pressure about an arbitrary value of $\rho$, and that, moreover, an equation of state $p=p(\rho)$ must become linear as $p \rightarrow \infty$ in order that the sound speed $\sqrt{p^{\prime}(\rho)}$ not exceed the speed of light at large densities $\rho$ (see Sect. 3).

In this paper we will analyze the case when the equation of state is given by $p=\sigma^{2} \rho$, where the sound speed $\sigma$ is assumed to be constant. (Note that $\sigma$ has the dimensions of velocity because we take $\rho$ to have the dimensions of mass, not energy.) We note that, whereas in the classical regime the equation of state $p=\sigma^{2} \rho$ can only be viewed as a model problem for fluid flow, in the relativistic regime, this equation of state is of fundamental importance. It is also interesting to note that the hyperbolic conservation laws (1) play a more basic role in relativistic fluids than in classical fluids because infinite speed of propagation, which is a property of parabolic type systems such as the Navier-Stokes equations, is ruled out at the start in this setting by the relativistic principle that all velocities are bounded by $c$.

We study here the initial value problem for (1) in a two dimensional spacetime $\left(x^{0}, x^{1}\right)$, so that $\rho$ and $u$ are unknown functions of $\left(x^{0}, x^{1}\right)$, and

$$
\eta^{i j}=\left[\begin{array}{rr}
-1 & 0 \\
0 & 1
\end{array}\right] \text {. }
$$

Under these assumptions the stress-energy tensor (2) takes the form

$$
T^{i j}=\left[\begin{array}{cc}
\left(p+\rho c^{2}\right) u^{0} u^{0}-p & \left(p+\rho c^{2}\right) u^{0} u^{1} \\
\left(p+\rho c^{2}\right) u^{0} u^{1} & \left(p+\rho c^{2}\right) u^{1} u^{1}+p
\end{array}\right] .
$$

In one space and one time dimension, the system (1), $\operatorname{div} T=0$, provides a model for the dynamics of plane waves in special relativistic fluids.

For our theorem we assume that $p$ and $\rho$ satisfy an equation of state of the form

$$
p=\sigma^{2} \rho,
$$

where $\sigma^{2}$, the sound speed, is taken to be constant, $\sigma<c$. In particular, when $\sigma^{2}=c^{2} / 3$, (6) gives the important relativistic case $p=\left(c^{2} / 3\right) \rho$ discussed above. Since the background metric is the flat Minkowski metric $\eta_{i j}$, the increment of proper time $\tau$, (Minkowski arclength), along a curve is given by the formula

$$
d(\xi)^{2}=-\eta_{i j} d x^{i} d x^{j}
$$

${ }^{4}$ We thank D. Christodoulou for bringing this to our attention, [2] 
where we use the notation $\xi=c \tau$. In this way the coordinate time $t$ and the proper time $\tau$ have the dimensions of time, while $x^{0}$ and $\xi$ have the dimensions of length. Since $u=d x^{i} / d(c \tau)$, (where differentiation is taken along a particle path), defines the dimensionless velocity of the fluid, we must have

$$
u^{0}=\sqrt{1+\left(u^{1}\right)^{2}}
$$

Thus letting $u \equiv u^{1}$, the equations we consider are

$$
\begin{array}{r}
\frac{\partial}{\partial x^{0}}\left\{\left(p+\rho c^{2}\right)\left(1+u^{2}\right)-p\right\}+\frac{\partial}{\partial x^{1}}\left\{\left(p+\rho c^{2}\right) u \sqrt{1+u^{2}}\right\}=0, \\
\frac{\partial}{\partial x^{0}}\left\{\left(p+\rho c^{2}\right) u \sqrt{1+u^{2}}\right\}+\frac{\partial}{\partial x^{1}}\left\{\left(p+\rho c^{2}\right) u^{2}+p\right\}=0,
\end{array}
$$

together with the initial data

$$
\rho\left(0, x^{1}\right)=\rho_{0}\left(x^{1}\right), \quad u\left(0, x^{1}\right)=u_{0}\left(x^{1}\right) .
$$

Equations (9) form a system of nonlinear hyperbolic conservation laws in the sense of Lax [7]. Thus if one seeks global (in time) solutions, then due to the formation of shock waves, one must extend the notion of solution, in the usual way, [16], in order to admit as solutions such as discontinuous functions.

In the classical limit, the relativistic system (9) reduces to the classical version of the compressible Euler equations. In order to observe this correspondence throughout, we set $x \equiv x^{1}$, choose $\mathbf{x} \equiv(x, t)$ as the independent variables, and replace the invariant velocity $u$ in system (9) in favor of its expression in terms of the classical coordinate velocity $v \equiv d x / d t$ of the particle paths of the fluid. To accomplish this, note that by (8),

$$
\frac{d t}{d \tau}=\frac{d x^{0}}{d \xi}=\sqrt{1+u^{2}}
$$

so we can write

$$
v=\frac{d x^{1}}{d t}=\frac{d x^{1}}{d \xi} \frac{d \xi}{d t}=c u \sqrt{1+u^{2}}
$$

which solving for $u$ gives

$$
u=v / \sqrt{c^{2}-v^{2}} .
$$

The mapping $u \rightarrow v$ in (12) defines a smooth 1-1 mapping from $(-\infty,+\infty)$ to $(-c, c)$, and so there is no loss of generality in taking $v$ as the state variable instead of $u$.

Now writing system (9) in terms of $\rho$ and $v$ and multiplying the first equation by $1 / c$, we obtain the general system

$$
\begin{aligned}
& \frac{\partial}{\partial t}\left\{\frac{\left(p+\rho c^{2}\right)}{c^{2}} \frac{v^{2}}{c^{2}-v^{2}}+\rho\right\}+\frac{\partial}{\partial x}\left\{\left(p+\rho c^{2}\right) \frac{v}{c^{2}-v^{2}}\right\}=0, \\
& \frac{\partial}{\partial t}\left\{\left(p+\rho c^{2}\right) \frac{v^{2}}{c^{2}-v^{2}}\right\}+\frac{\partial}{\partial x}\left\{\left(p+\rho c^{2}\right) \frac{v^{2}}{c^{2}-v^{2}}+p\right\}=0 .
\end{aligned}
$$


Restricting to the case $p=\sigma^{2} \rho,(13)$ reduces to

$$
\begin{aligned}
& \frac{\partial}{\partial t}\left\{\rho\left[\left(\frac{\sigma^{2}+c^{2}}{c^{2}}\right) \frac{v^{2}}{c^{2}-v^{2}}+1\right]\right\}+\frac{\partial}{\partial x}\left\{\rho\left[\left(\sigma^{2}+c^{2}\right) \frac{v^{2}}{c^{2}-v^{2}}\right]\right\}=0, \\
& \frac{\partial}{\partial t}\left\{\rho\left[\left(\sigma^{2}+c^{2}\right) \frac{v}{c^{2}-v^{2}}\right]\right\}+\frac{\partial}{\partial x}\left\{\rho\left[\left(\sigma^{2}+c^{2}\right) \frac{v^{2}}{c^{2}-v^{2}}+\sigma^{2}\right]\right\}=0,
\end{aligned}
$$

together with the initial conditions

$$
\rho(x, 0)=\rho_{0}(x), \quad v(x, 0)=v_{0}(x) .
$$

Note that in the limit $c \rightarrow \infty$, the system (13) reduces to the classical system

$$
\begin{gathered}
\rho_{t}+(\rho v)_{x}=0, \\
(\rho v)_{t}+\left(\rho v^{2}+\sigma^{2} \rho\right)_{x}=0 .
\end{gathered}
$$

The main purpose of this paper is to prove the following theorem:

Theorem 1. Let $\rho_{0}(x)$ and $v_{0}(x)$ be arbitrary initial data satisfying

$$
\operatorname{Var}\left\{\ln \left(\rho_{0}(\cdot)\right)\right\}<\infty,
$$

and

$$
\operatorname{Var}\left\{\ln \left(\frac{c+v_{0}}{c-v_{0}}\right)\right\}<\infty,
$$

where $\operatorname{Var}\{f(\cdot)\}$ denotes the total variation of the function $f(x), x \in \mathbf{R}$. Then there exists a bounded weak solution $(\rho(x, t), u(x, t))$ of $(14)$ satisfying

$$
\operatorname{Var}\{\ln (\rho(\cdot, t))\} \leqq V_{0},
$$

and

$$
\operatorname{Var}\left\{\ln \left(\frac{c+v(\cdot, t)}{c-v(\cdot, t)}\right)\right\} \leqq V_{1},
$$

where (19) and (20) are Lorentz invariant statements, and $V_{0}$ and $V_{1}$ are Lorentz invariant constants depending only on the initial total variation bounds assumed in (17) and (18). Moreover, the solution is a limit of approximate solutions $\left(\rho_{\Delta x}, u_{\Delta x}\right)$ which satisfy the "energy inequality"

$$
\operatorname{Var}\left\{\ln \left(\rho_{\Delta x}(t+, \cdot)\right)\right\} \leqq \operatorname{Var}\left\{\ln \left(\rho_{\Delta x}(s+, \cdot)\right)\right\},
$$

for all times $0 \leqq s \leqq t$. The approximate solutions are generated by Glimm's method [5], and converge pointwise a.e., and in $L_{\mathrm{loc}}^{1}$ at each time, uniformly on bounded subsets of $(x, t)$-space.

In one space dimension, the total variation of a solution at a fixed time $t \geqq 0$ is a natural measure of the total wave strength present in the solution at time $t$. The non-increasing property of $\ln (\rho)$ is a very special property of system (14), there being no way to construct such a function for a general $2 \times 2$ system of conservation laws [5]. We conjecture that the inequality (21) is valid for the weak solutions themselves, i.e., that

$$
\operatorname{Var}\{\ln (\rho(\cdot, t+))\} \leqq \operatorname{Var}\{\ln (\rho(\cdot, s+))\},
$$


for all $s \leqq t$. Such an inequality would provide a Lorentz invariant monotonicity property of the weak solutions of (14) that refines the estimate (19).

To prove Theorem 1, we develop an analysis which parallels that first given by Nishida (1968) in [12] for the classical system (16). Nishida's result provided the first "big data" global existence theorem for weak solutions of the classical compressible Euler equations, and it remains the only argument for stability of solutions in a derivative norm that applies to arbitrarily large initial data. (Nishida originally treated the Lagrangian formulation of system (16), [3, 16]. A Lagrangian formulation of the relativistic model can be found in [17].) Theorem 1 shows (surprisingly!) that the ideas of Nishida generalize to the relativistic case (14) where the equations are significantly more complicated. Indeed, the special properties of the system (14) that lead to the estimates (19) and (20) require not only that $p$ be linear in $\rho$, but are also highly dependent on the specific form of the velocity terms; these appearing in a different and more complicated form in the relativistic equations (14) than in the classical equations (16). The technique of Nishida is to analyze solutions via the Glimm difference scheme [5] through an analysis of wave interactions in the plane of Riemann invariants. The main technical point in his analysis involves showing that the shock curves based at different points are congruent in the plane of Riemann invariants. We show that this property carries over to the relativistic case by obtaining a new global parameterization of the shock curves. Of course, in the relativistic case, the shock curves are given by considerably more complicated functions. Our analysis exploits the Lorentz invariance properties of system (14), and thus we shall take care to develop the geometric properties of the constructions used in our analysis.

Note that if we non-dimensionalize systems (14) and (16) by multiplying through by the appropriate powers of $c$ and replacing $\partial / \partial t$ in terms of $\partial / \partial x^{0}$, we obtain two systems in the variables $\rho$ and $v / c$, each parameterized by the dimensionless quantity $\sigma / c$. Thus we can say that Theorem 1 and Nishida's result [12] establish a "large data" existence theorem for the two distinct one parameter families of dimensionless systems which correspond to (14) and (16). But note also that system (16) is obtained by taking the limit $c \rightarrow \infty$ in (14), and thus we can obtain the congruence property of the shock curves for (16) by applying the limit $c \rightarrow \infty$ to our formulas for (14), and in this sense we can view Theorem 1 as a generalization of Nishida's theorem [12]. ${ }^{5}$ This is done at the end of Sect. 5.

In his original paper [12], Nishida did not actually obtain the result that the invariant quantity, $\operatorname{Var}\{\ln (\rho)\}$, is non-increasing on approximate solutions. The idea for (21) in Nishida's case came from Liu [8], and a similar idea was exploited by Luskin and Temple in [9]; see also [13].

The organization of this paper is as follows: In Sect. 2 we put the problem (14), (15) in the context of the general theory of conservation laws, prove the regularity of the mapping from the plane of conserved quantities to the $(\rho, v)$-plane, and we show that (19) and (20) are Lorentz invariant statements. In Sect. 3 we use the Rankine Hugoniot jump relations to derive the wave speeds $\lambda_{i}$ and Riemann invariants for (13) in the case of a general barotropic equation of state $p=p(\rho)$. In this general setting, we shall also derive necessary and sufficient conditions (on the function $p(\rho)$ ) for the system (14) to be strictly hyperbolic and genuinely nonlinear in the sense of Lax $[7,16]$. We note that the assumption that wave speeds are

${ }^{5}$ We thank J. Rauch for pointing this out 
bounded by $c$ imposes a linear growth rate on $p(\rho)$ as $\rho \rightarrow \infty$, and thus there is a possibility of losing genuine nonlinearity of the system in this limit. Thus, in Sect. 3 we describe the properties of what we call the relativistic $p$-system. In Sects. 4-7 we restrict to the case $p=\sigma^{2} \rho$ and develop the geometry of the shock curves in Riemann-invariant space, solve the Riemann problem, and use the Glimm difference scheme to prove Theorem 1. In the appendix we derive the transformation properties of the Rankine Hugoniot jump relations for general relativistic conservation laws. The analysis applies to arbitrary nonlinear spacetime coordinate transformations in 4-dimensional spacetime with arbitrary Lorentzian spacetime metric. We use this to give a simple derivation of the covariance properties of the characteristics, and the transformation formulas for the characteristic speeds and shock speeds in 2-dimensional special relativity.

\section{Systems of Conservation Laws}

In this section we put the problem $(14),(15)$ in the context of the general theory of conservation laws, and discuss the Lorentz invariant properties of the system.

The problem (14) and (15) is a special case of the initial value problem for a general system of nonlinear hyperbolic conservation laws in the sense of Lax $[7,16]$,

$$
\begin{aligned}
& U_{t}+F(U)_{x}=0, \\
& U(x, 0)=U_{0}(x),
\end{aligned}
$$

where in our case

$$
U \equiv\left(\rho\left[\frac{\left(\sigma^{2}+c^{2}\right)}{c^{2}} \frac{v^{2}}{c^{2}-v^{2}}+1\right], \rho\left(\sigma^{2}+c^{2}\right) \frac{v}{c^{2}-v^{2}}\right)
$$

and

$$
F(U) \equiv\left(\rho\left(\sigma^{2}+c^{2}\right) \frac{v}{c^{2}-v^{2}}, \rho\left[\left(\sigma^{2}+c^{2}\right) \frac{v^{2}}{c^{2}-v^{2}}+\sigma^{2}\right]\right) .
$$

In order to apply Glimm's method (cf. Sect. 7), we need the following result.

Proposition 1. The mapping $(\rho, v) \rightarrow\left(U_{1}, U_{2}\right)=U$ is $1-1$, and the Jacobian determinant of this mapping is both continuous and non-zero in the region $\rho>0,|v|<c$.

Proof. If the mapping were not $1-1$, then there would be points $(\rho, v),(\bar{\rho}, \bar{v})$ such that $U(\rho, v)=U(\bar{\rho}, \bar{v})$. Since $\frac{\partial U_{i}}{\partial \rho} \neq 0$ for $i=1,2$, we may assume that $v \neq \bar{v}$. Now using (25), we have

$$
\frac{\rho}{\bar{\rho}}\left\{\frac{\sigma^{2}+c^{2}}{c^{2}} \frac{v^{2}}{c^{2}-v^{2}}+1\right\}=\left\{\frac{\sigma^{2}+c^{2}}{c^{2}} \frac{\bar{v}^{2}}{c^{2}-\bar{v}^{2}}+1\right\},
$$

and

$$
\frac{\rho}{\bar{\rho}}\left\{\frac{v}{c^{2}-v^{2}}\right\}=\frac{\bar{v}}{c^{2}-\bar{v}^{2}}
$$


Eliminating $\frac{\rho}{\bar{\rho}}$ and simplifying gives

$$
(v-\bar{v})\left\{\frac{\sigma^{2}}{c^{2}} v \bar{v}-c^{2}\right\}=0
$$

Since $v \neq \bar{v}$, this implies

$$
\frac{\sigma^{2}}{c^{2}} v \bar{v}-c^{2}=0
$$

which contradicts the assumptions $|\sigma|<c,|v|<c$ and $|\bar{v}|<c$. Thus the mapping is $1-1$. A straightforward calculation shows that

$$
\operatorname{det}\left(\frac{\partial\left(U_{1}, U_{2}\right)}{\partial(\rho, v)}\right)=\frac{\rho\left(\sigma^{2}+c^{2}\right)}{c^{2}\left(c^{2}-v^{2}\right)^{2}}\left\{c^{4}-v^{2} \sigma^{2}\right\}>0 \text {. }
$$

It is important to note that the systems (13) and (14) are Lorentz invariant. This means that under any Lorentz transformation $(t, x) \rightarrow(\bar{t}, \bar{x})$, one obtains an identical system in the barred coordinates once the velocity states are renamed in terms of the coordinate velocities as measured in the barred coordinate system. Thus, in particular, under Lorentz transformations, $\rho(t, x)$ is a scalar invariant, and thus it takes the same value in the barred and unbarred coordinates that name the same geometric point in the background spacetime manifold. On the other hand, the velocity $v$ is not a scalar, since it is formed from the entries of the vector quantity $\left(u^{0}, u^{1}\right)$. In this paper we will exploit the transformation law for velocities by calculating the shock curves and shock speeds in a frame in which the particle velocity $v$ is zero, and then applying the Lorentz transformation law for velocities to obtain these quantities in an arbitrary frame. The velocity transformation law can be given as follows (cf. [20]): If in a Lorentz transformation, the barred frame $(\bar{t}, \bar{x})$ moves with velocity $\mu$ as a measured in the unbarred frame $(t, x)$, and if $v$ denotes the velocity of a particle as measured in the unbarred frame, and $\bar{v}$ the velocity of the same particle as measured in the barred frame, then

$$
v=\frac{\mu+\bar{v}}{1+\frac{\mu \bar{v}}{c^{2}}}
$$

Since under Lorentz transformations $\rho$ transforms like a scalar but $v$ does not, it follows that the estimate (19), which is based on the scalar $\rho$ and not the velocity $v$, expresses a Lorentz invariant property of the weak solutions of (13), (14). On the other hand, the estimate (20) is based on the quantity $\ln \left(\frac{c+v}{c-v}\right)$, which is not a Lorentz invariant scalar quantity. Nevertheless, it turns out, (remarkably!), that $\operatorname{Var}\left\{\ln \left(\frac{c+v(\cdot, t)}{c-v(\cdot, t)}\right)\right\}$ is Lorentz invariant, and thus (20), as well as (19), is a Lorentz invariant statement. This is a consequence of the following result.

Proposition 2. Let $v(x, t)$ be any velocity field which satisfies the velocity transformation law (27) under Lorentz transformations. Then

$$
\operatorname{Var}_{x}\left\{\ln \left(\frac{c+v(x, t)}{c-v(x, t)}\right)\right\}=\operatorname{Var}_{x}\left\{\ln \left(\frac{c+\bar{v}(L(x, t))}{c-\bar{v}(L(x, t))}\right)\right\},
$$

where $L$ is any Lorentz transformation, $\overline{\mathbf{x}}=L \mathbf{x}$, and $v$ and $\bar{v}$ are related by (27). 
Proof. By (27), $v$ and $\bar{v}$ are related by the equation

$$
v(x, t)=\frac{\mu+\bar{v}(L(x, t))}{1+\frac{\mu \bar{v}(L(x, t))}{c^{2}}},
$$

where $\mu$ is the velocity of the barred frame $\overline{\mathbf{x}}$ as measured in the unbarred frame $\mathbf{x}$. Then for any $x_{i-1}<x_{i}$, this implies

$$
\begin{aligned}
\ln \left\{\frac{c+v\left(x_{i}, t\right)}{c-v\left(x_{i}, t\right)}\right\}-\ln \left\{\frac{c+v\left(x_{i-1}, t\right)}{c-v\left(x_{i-1}, t\right)}\right\}=\ln \left\{\left(\frac{c+v\left(x_{i}, t\right)}{c-v\left(x_{i}, t\right)}\right)\left(\frac{c+v\left(x_{i-1}, t\right)}{c-v\left(x_{i-1}, t\right)}\right)\right\} \\
=\ln \left\{\left(\frac{c+\bar{v}\left(x_{i}, t\right)}{c-\bar{v}\left(x_{i}, t\right)}\right)\left(\frac{c+\bar{v}\left(x_{i-1}, t\right)}{c-\bar{v}\left(x_{i-1}, t\right)}\right)\right\} \\
=\ln \left\{\frac{c+v\left(x_{i}, t\right)}{c-v\left(x_{i}, t\right)}\right\}-\ln \left\{\frac{c+v\left(x_{i-1}, t\right)}{c-v\left(x_{i-1}, t\right)}\right\},
\end{aligned}
$$

from which (28) follows.

\section{The Wave Speeds}

In this section we construct the eigenvalues and Riemann invariants that are associated with the system of conservation laws (14).

First recall the three important velocities associated with a system (14): the particle velocity $v$, the wave speeds $\lambda_{i}(\rho, v)$ and the shock speeds $s_{i}(\rho, v), i=1,2$. The wave speeds are the speeds of propagation of the characteristic curves, and for (23), the $\lambda_{i}$ are the eigenvalues of the $2 \times 2$ matrix of derivatives $d F \equiv \partial F / \partial U$. Thus $d F R_{i}=\lambda_{i} R_{i}$, where $R_{i}$ denotes the $i^{\text {th }}$ right eigenvector of $d F$. For weak solutions of (23), discontinuities propagate at the shock speeds $s_{i}$ which are determined from the Rankine-Hugoniot jump relations (see [7])

$$
s[U]=[F] .
$$

Here $[f] \equiv f_{L}-f_{R}$ denotes the jump in the function $f(U)$ between the left and right hand states along the curve of discontinuity in the $x t$-plane. It is not a priori clear that a characteristic curve or shock curve $(x(\tau), t(\tau))$, computed in one Lorentz coordinate system will transform to the same spacetime curve when computed in a different Lorentz frame. In the Appendix we will show that both properties are a consequence of the conservation form of the equations. It follows that the derivative $\left(x^{\prime}(\tau), t^{\prime}(\tau)\right)$ transforms like a vector field and that the corresponding speed $x^{\prime}(\tau) / t^{\prime}(\tau)$ transforms by the relativistic transformation law for velocities. Thus we can conclude that $\lambda_{i}$ and $s_{i}$ transform according to (27) under a Lorentz transformation. Since in the system (14), the flux $F$ is given implicitly as a rather complicated function of $U$, it is convenient to note that $\lambda_{i}, R_{i}$, and $s_{i}$ can all be calculated from the jump relation (29) alone. For this we need the following well-known theorem due to $\operatorname{Lax},[7,16]$ :

Theorem 2. Assume that the system (23) is strictly hyperbolic; i.e., that $\lambda_{1}<\lambda_{2}$ in the physical domain of $U$. Then, for a fixed state $U_{L}$, the solutions $U$ and $s$ of the Rankine-Hugoniot relation $s\left(U-U_{L}\right)=F(U)-F\left(U_{L}\right)$ can be described (in 
a neighborhood of $\left.U_{L}\right)$ by two families of smooth curves $U=\mathbf{S}_{\mathrm{i}}(\varepsilon), \mathbf{S}_{\mathrm{i}}(0)=U_{L}$, with corresponding speeds $s_{i}(\varepsilon), i=1,2$. Moreover, as $\varepsilon \rightarrow 0$, we have $s_{i}(\varepsilon) \rightarrow \lambda_{i}\left(U_{L}\right)$ and $U_{i}^{\prime}(\varepsilon) \rightarrow R_{i}$. Here, the parameter $\varepsilon$ can be taken to be (Euclidean) arclength along the shock curve $\mathbf{S}_{\mathbf{i}}$ in $U$-space.

We now use Theorem 2 to obtain the eigenpairs $\left(\lambda_{i}, R_{i}\right), i=1,2$, for system (14) in the case of a general equation of state of the form $p=p(\rho)$. To start, write system (14) in the form

$$
\begin{aligned}
& A_{t}+B_{x}=0, \\
& B_{t}+C_{x}=0,
\end{aligned}
$$

where

$$
\begin{aligned}
& A=\frac{\left(p+\rho c^{2}\right)}{c^{2}} \frac{v^{2}}{c^{2}-v^{2}}+\rho, \\
& B=\left(p+\rho c^{2}\right) \frac{v}{c^{2}-v^{2}}, \\
& C=\left(p+\rho c^{2}\right) \frac{v^{2}}{c^{2}-v^{2}}+p .
\end{aligned}
$$

Then by (29), for fixed $\left(\rho_{L}, v_{L}\right)$, the state $(\rho, v) \equiv\left(\rho_{R}, v_{R}\right)$ lies on a shock curve if and only if

$$
[B]^{2}=[A][C] \text {, }
$$

where, for example, $[A] \equiv A-A_{L}$ and $A \equiv A(\rho, v)$ is a function of the unknowns $\rho$ and $v$ along the shock curve. Now assuming that (32) defines $v$ implicitly as a function of $\rho$, (this assumption is justified by the construction of the solution itself) differentiate (32) with respect to $\rho$ and divide by $[B]$ to obtain

$$
2 B^{\prime}=\frac{[A]}{[B]} C^{\prime}+\frac{[C]}{[B]} A^{\prime},
$$

where prime denotes $d / d \rho$. We first obtain a formula for $d v / d \rho$ evaluated at $\rho=\rho_{L}$. To this end, note that by L'Hospital's rule,

$$
\lim _{\rho \rightarrow \rho_{L}} \frac{[A]}{[B]}=\frac{A^{\prime}}{B^{\prime}},
$$

where the right-hand side is evaluated at $\rho=\rho_{L}$. Thus, at $\rho=\rho_{L}$, Eq. (33) reduces to

$$
\left(B^{\prime}\right)^{2}=A^{\prime} C^{\prime} \text {. }
$$

Using (31) we find

$$
\begin{aligned}
& A^{\prime}=\frac{\left(p^{\prime}+c^{2}\right)}{c^{2}} e+\frac{\left(p+\rho c^{2}\right)}{c^{2}} \frac{d e}{d v} v^{\prime}+1, \\
& B^{\prime}=\left(p^{\prime}+c^{2}\right) \frac{e}{v}+\left(p+\rho c^{2}\right) \frac{\left(v \frac{d e}{d v}-e\right) v^{\prime}}{v^{2}}, \\
& C^{\prime}=\left(p^{\prime}+c^{2}\right) e+\left(p+\rho c^{2}\right) \frac{d e}{d v} v^{\prime}+p^{\prime},
\end{aligned}
$$


where

$$
\begin{gathered}
e \equiv \frac{v^{2}}{c^{2}-v^{2}}, \\
\frac{d e}{d v}=\frac{2 c^{2} v}{c^{2}-v^{2}},
\end{gathered}
$$

and all terms are evaluated at $\rho=\rho_{L}$. Substituting (35) into (34) and collecting like powers of $v^{\prime}$ yields

$$
\begin{aligned}
0= & \left(v^{\prime}\right)^{2}\left\{\left(p+\rho c^{2}\right)^{2} \frac{\left(v \frac{d e}{d v}-e\right)^{2}}{v^{4}}-\frac{\left(p+\rho c^{2}\right)^{2}}{c^{2}}\left(\frac{d e}{d v}\right)^{2}\right\}_{1} \\
& +v^{\prime}\left\{\frac{2}{v^{3}}\left(p^{\prime}+c^{2}\right)\left(p+\rho c^{2}\right) e\left(v \frac{d e}{d v}-e\right)\right. \\
& \left.-\frac{\left(p+\rho c^{2}\right)}{c^{2}} e^{\prime}\left(p^{\prime}+2\left(p^{\prime}+c^{2}\right) e+1\right)\right\}_{2} \\
& +\left\{\left(\left(p^{\prime}+c^{2}\right)^{2} \frac{e^{2}}{v^{2}}-\frac{\left(p^{\prime}+c^{2}\right) e}{c^{2}}+1\right)\left(\left(p^{\prime}+c^{2}\right) e+p^{\prime}\right)\right\}_{3} .
\end{aligned}
$$

Here we label the brackets so that we can evaluate them separately. A calculation using (36) and (37) in (38) gives

$$
\begin{aligned}
& \{\cdot\}_{1}=\frac{\left(p+\rho c^{2}\right)^{2}}{\left(c^{2}-v^{2}\right)^{2}}, \\
& \{\cdot\}_{2}=0, \\
& \{\cdot\}_{3}=-p^{\prime} .
\end{aligned}
$$

Substituting these into (38) we conclude

$$
\frac{v^{\prime}}{c^{2}-v^{2}}= \pm \frac{\sqrt{p^{\prime}}}{p+\rho c^{2}} .
$$

We can now solve for the Riemann invariants associated with system (14). Recall that a Riemann invariant for (23) is a scalar function $f, \nabla f \neq 0$, which is constant along the integral curves of one of the eigenvector fields of matrix field $d F$. By Theorem 2 , the shock curves $S_{i}$ are tangent to the eigenvectors $R_{i}$ at $\rho=\rho_{L}$, and thus the Riemann invariants for system (14) satisfy the differential equations

$$
\frac{d v}{d \rho}= \pm \frac{\sqrt{p^{\prime}}\left(c^{2}-v^{2}\right)}{p+\rho c^{2}}
$$

which have the solutions

$$
\frac{1}{2} \ln \left(\frac{c+v}{c-v}\right)= \pm c \int \frac{\sqrt{p^{\prime}(s)}}{p(s)+c^{2} s} d s .
$$


Thus we may define a pair of Riemann invariants $r$ and $s$ for system (14) as

$$
\begin{aligned}
& r=\frac{1}{2} \ln \left(\frac{c+v}{c-v}\right)+c \int_{1}^{\rho} \frac{\sqrt{p^{\prime}(s)}}{p(s)+c^{2} s} d s, \\
& s=\frac{1}{2} \ln \left(\frac{c+v}{c-v}\right)-c \int_{1}^{\rho} \frac{\sqrt{p^{\prime}(s)}}{p(s)+c^{2} s} d s .
\end{aligned}
$$

We now calculate the eigenvalues $\lambda_{i}(\rho, v)$ of system (14). By Theorem 2 , these are obtained as the limit of $s$ in (29) as $\rho \rightarrow \rho_{L}$. Solving for $s$ in (30) we obtain

$$
s[A]=[B] .
$$

Thus, again assuming that $v=v(\rho)$, Theorem 2 implies

$$
\begin{aligned}
\lambda_{i} & =\lim _{\rho \rightarrow \rho_{L}} \frac{[B]}{[A]} \\
& =\lim _{\rho \rightarrow \rho_{L}} c \frac{\left(p+\rho c^{2}\right) e+p}{\left(p+\rho c^{2}\right) e+\rho c^{2}} \\
& =\lim _{\rho \rightarrow \rho_{L}} c \frac{\left(p^{\prime}+c^{2}\right) e+\left(p+\rho c^{2}\right) \frac{d e}{d v} v^{\prime}+p^{\prime}}{\left(p^{\prime}+c^{2}\right) e+\left(p+\rho c^{2}\right) \frac{d e}{d v} v^{\prime}+c^{2}},
\end{aligned}
$$

where we have applied L'Hospital's rule. But from (3),

$$
\frac{d v}{d \rho}= \pm \frac{\sqrt{p^{\prime}}}{p+\rho c^{2}}\left(c^{2}-v^{2}\right),
$$

so substituting (44) into (43) and simplifying gives

$$
\lambda_{1}=\frac{v-\sqrt{p^{\prime}}}{1-\frac{v \sqrt{p^{\prime}}}{c^{2}}},
$$

and

$$
\lambda_{2}=\frac{v+\sqrt{p^{\prime}}}{1+\frac{v \sqrt{p^{\prime}}}{c^{2}}} .
$$

One can now verify directly that $r$ [resp. $s]$ is a 1- [resp. 2-] Riemann invariant for system (14), by which we mean that $r$ [resp. $s$ ] is constant along integral curves of $R_{2}$ [resp. $R_{1}$ ].

We now utilize the formulas (41), (42), (45) and (46) to obtain conditions on $p$ under which the system (13) is strictly hyperbolic and genuinely nonlinear in the sense of Lax [7]. Recall that a system of conservation laws (23) is said to be genuinely nonlinear in the $i^{\text {th }}$ characteristic family $\left(\lambda_{i}, R_{i}\right)$ if $\nabla_{U} \lambda_{i} \cdot R_{i} \neq 0$ at each point $U$ in state space. (Here, the $\nabla$ denotes the standard Euclidean gradient on state space $(\rho, v)$.) The following theorem gives necessary and sufficient conditions 
for system (14), with barotropic equation of state $p=p(\rho)$, to be strictly hyperbolic and genuinely nonlinear.

Theorem 3. System (13) is strictly hyperbolic at $(\rho, v)$ if and only if

$$
\sqrt{p^{\prime}(\rho)}<c .
$$

Moreover, assuming (47), system (13) is genuinely nonlinear at each $(\rho, v)$ if and only if

$$
p^{\prime \prime}(\rho) \geqq-2 \frac{\left(c^{2}-p^{\prime}\right) p^{\prime}}{p+\rho c^{2}} .
$$

Inequality (47) is also necessary and sufficient for the sound speeds $\lambda_{i}$ to be bounded by $c$. Moreover, note that the condition for genuine nonlinearity is a geometric condition, being a condition involving only a function of the scalar invariant $\rho$. Indeed, by Lorentz invariance we know a priori that the condition for genuine nonlinearity could not have involved the state variable $v$, for if it did, then we reach the absurd conclusion that a Lorentz change of frame could change the wave structure of solutions.

Proof. When $\sqrt{p^{\prime}(\rho)}=c, \lambda_{i}= \pm c$, so (47) is required for $|\lambda| \leqq c$. It is straightforward to verify that when $\sqrt{p^{\prime}(\rho)}<c, \lambda_{1}<\lambda_{2}$ holds. To verify (48), note that $r$ and $s$ are constant on integral curves of $R_{2}$ and $R_{1}$, respectively. Differentiating (41) and (42) gives

$$
\frac{\partial r}{\partial \rho}=\frac{-c \sqrt{p^{\prime}}}{p+\rho c^{2}}, \quad \frac{\partial r}{\partial v}=\frac{c}{c^{2}-v^{2}}
$$

and

$$
\frac{\partial s}{\partial \rho}=\frac{c \sqrt{p^{\prime}}}{p+\rho c^{2}}, \quad \frac{\partial s}{\partial v}=\frac{c}{c^{2}-v^{2}} .
$$

Thus, in $(\rho, v)$-coordinates, we can take $R_{i}$ to be defined by

$$
R_{1} \equiv\left(\frac{-c}{c^{2}-v^{2}}, \frac{c \sqrt{p^{\prime}}}{p+\rho c^{2}}\right)^{t}
$$

and

$$
R_{2} \equiv\left(\frac{c}{c^{2}-v^{2}}, \frac{c \sqrt{p^{\prime}}}{p+\rho c^{2}}\right)^{t} .
$$

Using these it is straightforward to verify that

$$
\nabla \lambda_{1} \cdot R_{1}=\frac{c^{2}}{\left(\sqrt{p^{\prime}} v-c^{2}\right)^{2}}\left\{\frac{\left(c^{2}-p^{\prime}\right) \sqrt{p^{\prime}}}{p+\rho c^{2}}+\frac{p^{\prime \prime}}{2 \sqrt{p^{\prime}}}\right\}>0
$$

and

$$
\nabla \lambda_{2} \cdot R_{2}=\frac{c^{2}}{\left(\sqrt{p^{\prime}} v+c^{2}\right)^{2}}\left\{\frac{\left(c^{2}-p^{\prime}\right) \sqrt{p^{\prime}}}{p+\rho c^{2}}+\frac{p^{\prime \prime}}{2 \sqrt{p^{\prime}}}\right\}>0
$$

if and only if (48) holds. $\square$ 
Note that from the formulas (49) and (50) it follows that $\rho$ decreases [resp. increases] and $v$ increases along the integral curves of $R_{1}$ [resp. $\left.R_{2}\right]$ in the direction of increasing $\lambda_{1}$ [resp. $\left.\lambda_{2}\right]$. Thus, the integral curves of $R_{i}$ give the oriented rarefaction curves for system (14), $[7,16]$.

\section{The Shock Curves}

In this section we restrict to the case $p=\sigma^{2} \rho$ and obtain global parameterizations of the shock curves $\mathbf{S}_{\mathrm{i}}$ and shock speeds $s_{i}$ which are valid for arbitrary $\rho_{L}$ and $v_{L}$. (Here we let $\mathbf{S}_{\mathrm{i}}$ denote the portion of $\mathbf{S}_{\mathrm{i}}(\varepsilon)$, defined in Theorem 2, which corresponds to shocks satisfying the Lax condition. Upon normalizing $R_{i}$ by $\nabla \lambda_{i} \cdot R_{i}>0$, we can assume without loss of generality that $S_{i}$ is given locally by $\varepsilon \leqq 0,[7,16]$.) We use our global parameterization to study the geometry of the shock curves in Riemann invariant coordinates $(r, s)$.

Lemma 1. Assume that $\left(\rho_{L}, v_{L}\right)$ and $(\rho, v) \equiv\left(\rho_{R}, v_{R}\right)$ satisfy the jump conditions (29) for system (14) with equation of state (6). Then the following relations hold:

$$
\frac{\rho}{\rho_{L}}=1+\beta\left\{1 \mp \sqrt{1+\frac{2}{\beta}}\right\} \equiv f_{\mp}(\beta),
$$

where

$$
\beta \equiv \beta\left(v, v_{L}\right)=\frac{\left(\sigma^{2}+c^{2}\right)^{2}}{2 \sigma^{2}} \frac{\left(v-v_{L}\right)^{2}}{\left(c^{2}-v^{2}\right)\left(c^{2}-v_{L}^{2}\right)} .
$$

The shock curve $S_{1}$ is given by (53) and parameterized by $\rho \geqq \rho_{L}$ when we take a minus sign in (53), and $S_{2}$ is given by (53) and parameterized by $\rho \leqq \rho_{L}$ when we take the plus sign.

Proof. For system (14), the Rankine-Hugoniot jump conditions (29) give

$$
s\left[\rho \frac{\left(\sigma^{2}+c^{2}\right)}{c^{2}} \frac{v^{2}}{c^{2}-v^{2}}+\rho\right]=\left[\rho\left(\sigma^{2}+c^{2}\right) \frac{v}{c^{2}-v^{2}}\right],
$$

and

$$
s\left[\rho\left(\sigma^{2}+c^{2}\right) \frac{v}{c^{2}-v^{2}}\right]=\left[\rho\left(\sigma^{2}+c^{2}\right) \frac{v^{2}}{c^{2}-v^{2}}+\rho \sigma^{2}\right] .
$$

Eliminating the shock speed $s$ and recalling $u^{2}=v^{2} /\left(c^{2}-v^{2}\right)$ gives

$$
\begin{aligned}
\left\{\frac{c \rho v}{c^{2}-v^{2}}-\frac{c \rho_{L} v_{L}}{c^{2}-v_{L}^{2}}\right\}^{2}= & \left\{\frac{\rho v^{2}}{c^{2}-v^{2}}+\frac{\rho c^{2}}{\sigma^{2}+c^{2}}-\frac{\rho_{L} v_{L}^{2}}{c^{2}-v_{L}^{2}}-\frac{\rho_{L} c^{2}}{\sigma^{2}+c^{2}}\right\} \\
& \times\left\{\frac{\rho v^{2}}{c^{2}-v^{2}}+\frac{\rho \sigma^{2}}{\sigma^{2}+c^{2}}-\frac{\rho_{L} v_{L}^{2}}{c^{2}-v_{L}^{2}}-\frac{\rho_{L} \sigma^{2}}{\sigma^{2}+c^{2}}\right\} .
\end{aligned}
$$


Now multiplying out gives

$$
\begin{aligned}
0= & \left(\frac{\rho-\rho_{L}}{\rho_{L}}\right)^{2}\left\{u^{4}+u^{2}-\frac{c^{2} u^{4}}{v^{2}}+\frac{\sigma^{2} c^{2}}{\left(\sigma^{2}+c^{2}\right)^{2}}\right\}_{1} \\
& +\left(\frac{\rho-\rho_{L}}{\rho_{L}}\right)\left\{2 u^{2}\left[u^{2}\right]+\left[u^{2}\right]-\frac{2 c^{2} u^{2}}{v}\left[\frac{u^{2}}{v}\right]\right\}_{2} \\
& +\left\{\left[u^{2}\right]^{2}-c^{2}\left[\frac{u^{2}}{v}\right]^{2}\right\}_{3} .
\end{aligned}
$$

Simplifying, we obtain

$$
\begin{aligned}
& \{\cdot\}_{1}=\frac{\sigma^{2} c^{2}}{\left(\sigma^{2}+c^{2}\right)^{2}}, \\
& \{\cdot\}_{2}=-\frac{c^{2}\left(v-v_{L}\right)^{2}}{\left(c^{2}-v^{2}\right)\left(c^{2}-v_{L}^{2}\right)},
\end{aligned}
$$

and

$$
\{\cdot\}_{3}=-\frac{c^{2}\left(v-v_{L}\right)^{2}}{\left(c^{2}-v^{2}\right)\left(c^{2}-v_{L}^{2}\right)}
$$

so that (55) becomes

$$
\begin{aligned}
0= & \left(\frac{\rho-\rho_{L}}{\rho_{L}}\right)^{2}\left\{\frac{\sigma^{2} c^{2}}{\left(\sigma^{2}+c^{2}\right)^{2}}\right\}_{1} \\
& -\left(\frac{\rho-\rho_{L}}{\rho_{L}}\right)\left\{\frac{c^{2}\left(v-v_{L}\right)^{2}}{\left(c^{2}-v^{2}\right)\left(c^{2}-v_{L}^{2}\right)}\right\}_{2} \\
& -\left\{\frac{c^{2}\left(v-v_{L}\right)^{2}}{\left(c^{2}-v^{2}\right)\left(c^{2}-v_{L}^{2}\right)}\right\}_{3} .
\end{aligned}
$$

Now substituting $\beta$ (given in (54)) in (56) and solving for $\left(\rho-\rho_{0}\right) / \rho_{0}$ yields (53).

From Theorem 1 together with (49) and (50), it follows that for the shock curve $\mathbf{S}_{\mathbf{i}}$, which is tangent to $R_{i}$ at $\rho=\rho_{L}$, we must take a plus sign for $i=1$ and a minus sign when $i=2$. Moreover, by Theorem 2 together with (51) and (52), the $S_{1}$ is parameterized when $\rho \geqq \rho_{L}$, and $S_{2}$ is parameterized when $\rho \leqq \rho_{L}$.

The general theory of conservation laws only guarantees that the "Lax shock conditions" hold on $\mathbf{S}_{\mathbf{i}}$ near $\rho=\rho_{L},[16]$. We will show that these actually hold all along the shock curves $\mathbf{S}_{\mathbf{i}}$. We state this as part of the following lemma.

Lemma 2. Let $p=\sigma^{2} \rho$. Then the shock speed $s_{i}$ is monotone all along the shock curve $\mathbf{S}_{\mathbf{i}}$ of system (14), $i=1,2$, and moreover, the following inequalities (Lax shock conditions) hold at each state $U_{R} \neq U_{L}$ on the shock curve $\mathbf{S}_{\mathrm{i}}$ :

$$
\lambda_{i}\left(\rho_{L}, v_{L}\right)<s_{i}<\lambda_{i}\left(\rho_{R}, v_{R}\right) .
$$

Proof. First, since the shock speeds $s_{i}$ and wave speeds $\lambda_{i}$ transform by the velocity transformation law (27) (see appendix), it suffices to verify (57) in the case $v_{L}=0$. In this case we set $\rho_{L}=\rho_{0}$. Note that by Lemma $1, v \leqq v_{L}=0$ all along both $S_{1}$ and 
$S_{2}$. We first obtain $s_{i}$ and $\lambda_{i}$ along $\mathbf{S}_{\mathbf{i}}$ in terms of the parameter $\beta$ defined in (54). So, assuming $p=\sigma^{2} \rho$, the jump conditions (29) applied to system (14) directly give

$$
s^{2}=\left\{\left(\frac{z}{z-1}\right) \frac{c^{2} v^{2}}{c^{2}-v^{2}}+\frac{c^{2} \sigma^{2}}{c^{2}+\sigma^{2}}\right\} /\left\{\left(\frac{z}{z-1}\right) \frac{v^{2}}{c^{2}-v^{2}}+\frac{c^{2}}{c^{2}+\sigma^{2}}\right\},
$$

where we have set

$$
z=\frac{\rho}{\rho_{0}} .
$$

Now it follows from (53) that

$$
\frac{z}{z-1}=\frac{1+\beta \mp \sqrt{\beta^{2}+2 \beta}}{\beta \mp \sqrt{\beta^{2}+2 \beta}},
$$

and from (54) that

$$
v^{2}=c^{2} K \frac{\beta}{1+K \beta},
$$

where we set

$$
K=\frac{2 \sigma^{2} c^{2}}{\left(\sigma^{2}+c^{2}\right)^{2}}
$$

Using these in (58) gives (after simplification)

$$
s^{2}=c^{2} \frac{f_{\mp}(\beta)+\frac{\sigma^{2}}{c^{2}}}{f_{\mp}(\beta)+\frac{c^{2}}{\sigma^{2}}},
$$

where

$$
f_{\mp}(\beta)=1+\beta \mp \sqrt{\beta^{2}+2 \beta} .
$$

Note that the plus/minus sign in the above formulas agrees with the plus/minus sign in (53). Thus, $S_{1}$, is parameterized by $0 \leqq \beta<\infty$ (starting at $\left(\rho_{L}, v_{L}\right)$ ) when we choose the plus sign in (62) and $S_{2}$ is parameterized by $0 \leqq \beta<\infty$ when we choose the minus sign in (62). Specifically, on $S_{1}$,

$$
s_{1}(\beta)=-c \sqrt{\frac{1+\beta+\sqrt{\beta^{2}+2 \beta}+\frac{\sigma^{2}}{c^{2}}}{1+\beta+\sqrt{\beta^{2}+2 \beta}+\frac{c^{2}}{\sigma^{2}}}},
$$

and on $S_{2}$,

$$
s_{2}(\beta)=-c \sqrt{\frac{1+\beta-\sqrt{\beta^{2}+2 \beta}+\frac{\sigma^{2}}{c^{2}}}{1+\beta-\sqrt{\beta^{2}+2 \beta}+\frac{c^{2}}{\sigma^{2}}}} .
$$


We now obtain a corresponding parameterization of $\lambda_{i}$ with respect to $\beta$ along $\mathrm{S}_{\mathrm{i}}$. In the case $p=\sigma^{2} \rho$, the formulas (45) and (46) give

$$
\lambda_{1}=\frac{v-\sigma}{1-\frac{\sigma v}{c^{2}}},
$$

and

$$
\lambda_{2}=\frac{v+\sigma}{1+\frac{\sigma v}{c^{2}}}
$$

Thus

$$
\lambda_{1}=c^{2} \frac{\sigma+\sqrt{c^{2} K \frac{\beta}{1+K \beta}}}{-\sigma \sqrt{c^{2} K \frac{\beta}{1+K \beta}}-c^{2}},
$$

and

$$
\lambda_{2}=c^{2} \frac{\sigma-\sqrt{c^{2} K \frac{\beta}{1+K \beta}}}{-\sigma \sqrt{c^{2} K \frac{\beta}{1+K \beta}}+c^{2}},
$$

where we have used $v \leqq 0$.

Now differentiating (61) gives (for $\beta \neq 0, \sigma<c$ )

$$
\frac{d s_{i}^{2}}{d \beta}=c^{2} \frac{\left(\frac{c^{2}}{\sigma^{2}}-\frac{\sigma^{2}}{c^{2}}\right) f_{\mp}^{\prime}(\beta)}{\left(f_{\mp}(\beta)+\frac{c^{2}}{\sigma^{2}}\right)^{2}} \neq 0,
$$

from which the monotonicity of shock speeds along the shock curves easily follows. The inequality $s_{i}<\lambda_{i}\left(\rho_{L}, 0\right)$ thus holds. It remains to show that $\lambda_{i}<s_{i}$ along the shock curve $\mathbf{S}_{\mathrm{i}}$. We do the case $i=1$. To this end, let $x \equiv \sigma / c$ and set $z \equiv$ $\rho / \rho_{0}=f_{-}(\beta)$. Substituting into (63) and (65) yields

$$
\begin{aligned}
s_{1}^{2}-\lambda_{1}^{2}= & c^{2} \frac{\left(x-\sqrt{\frac{\sqrt{K}(z-1)}{\sqrt{2 z+K(1-z)^{2}}}}\right)^{2}}{x^{2}\left(z+\frac{1}{x^{2}}\right)}\left\{\frac{x^{2}\left(z+x^{2}\right)}{\left(x-\sqrt{\frac{\sqrt{K}(z-1)}{\sqrt{2 z+K(1-z)^{2}}}}\right)^{2}}\right. \\
& \left.-\frac{z+\frac{1}{x^{2}}}{\left(\frac{1}{x}-\sqrt{\frac{\sqrt{K}(z-1)}{\sqrt{2 z+K(1-z)^{2}}}}\right)^{2}}\right\}
\end{aligned}
$$




$$
=c^{2} \frac{x^{-2}\left(z+\frac{1}{x^{2}}\right)^{-1}\left(x-\sqrt{\left.\frac{\sqrt{K}(z-1)}{\sqrt{2 z+K(1-z)^{2}}}\right)^{2} \sqrt{2 z+K(1-z)^{2}}}\right.}{\left(x \sqrt{2 z+K(1-z)^{2}}-\sqrt{K}(z-1)\right)^{2}\left(\frac{1}{x} \sqrt{2 z+K(1-z)^{2}}-\sqrt{K}(z-1)\right)^{2}}\{\}_{*}
$$

where

$$
\begin{aligned}
\{\cdot\}_{*}= & \left(x^{2}\left(z+x^{2}\right)\right)\left(\frac{1}{x} \sqrt{2 z+K(1-z)^{2}}-\sqrt{K}(z-1)\right)^{2} \\
& -\left(z+\frac{1}{x^{2}}\right)\left(x \sqrt{2 z+K(1-z)^{2}}-\sqrt{K}(z-1)\right)^{2} .
\end{aligned}
$$

Thus it suffices to show that $\{\cdot\}_{*}<0$. A calculation using the identities

$$
\frac{x^{4}+x^{2}+1}{x^{2}}=\frac{2}{K}-1 \text {, }
$$

and

$$
\frac{x^{2}+1}{x}=\frac{2}{K}
$$

leads to

$$
\{\cdot\}_{*}=\left(1-x^{2}\right)(1-z)\left\{\frac{2}{K}+\frac{2^{3 / 2}}{K} \sqrt{2 z+K(1-z)^{2}}\right\} .
$$

But by (71) it follows that $\{\cdot\}<0$ for $z>1$, and thus by (70) we must have $s_{1}^{2}-\lambda_{1}^{2}<0$. Since both $s_{1}<0$ and $\lambda_{1}<0$ along $S_{1}$ when $v_{L}=0$, it follows that $\lambda_{1}<s_{1}$ all along $S_{1}$, thus finishing the proof of Lemma 2 .

\section{Geometry of the Shock Curves}

In this section we study the special geometry of the shock curves in the plane of Riemann invariants for system (14), the case $p=\sigma^{2} \rho$. In this case the shock curves are given by (53) and (54), and using (41) and (42), the Riemann invariants $r$ and $s$ are given in this case by (see Fig. 1)

$$
\begin{aligned}
& r=\frac{1}{2} \ln \left(\frac{c+v}{c-v}\right)-\sqrt{\frac{K}{2}} \ln (\rho), \\
& s=\frac{1}{2} \ln \left(\frac{c+v}{c-v}\right)+\sqrt{\frac{K}{2}} \ln (\rho),
\end{aligned}
$$

where $K$ is defined in (60).

Our main result of this section is that the $i$-shock curves are independent of the base point $\left(\rho_{L}, v_{L}\right)$ in the sense that, when graphed in the $r s$-plane, all $i$-shock curves are rigid translations of one another; and moreover, the 1-shock curve based at a given point is the reflection of the 2 -shock curve based at the same point about 


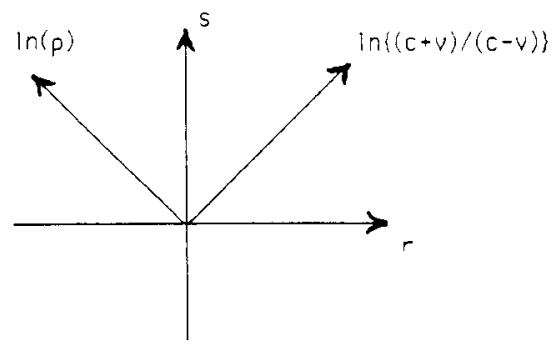

Fig. 1.

an appropriate axis of rotation. On an algebraic level, this happens because, along a shock curve, $\rho \rho_{L}^{-1}$ and $\left\{\frac{c+v}{c-v}\right\}\left\{\frac{c+v_{L}}{c-v_{L}}\right\}^{-1}$ in the definitions of $r$ and $s$ turn out to be functions of the parameter $\beta$ alone, and the functions that give $\rho \rho_{L}^{-1}$ as a function of $\beta$ are reciprocal on the 1- and 2-shock curves, respectively. We begin with the following lemma, which gives a parameterization of the $i$-shock curves for system (14) in the $r s$-plane:

Lemma 3. Let $r \equiv r(\rho, v), s \equiv s(\rho, v), \Delta r \equiv r(\rho, v)-r\left(\rho_{L}, v_{L}\right)$ and $\Delta s \equiv s(\rho, v)-$ $s\left(\rho_{L}, v_{L}\right)$, where we let $(\rho, v) \equiv\left(\rho_{R}, v_{R}\right)$. Then the 1-shock curve $S_{1}$ for system (14) based at $\left(r_{L}, s_{L}\right)$ is given by the following parameterization with respect to the parameter $\beta, 0 \leqq \beta<\infty$ :

$$
\begin{aligned}
& \Delta r=-\frac{1}{2} \ln \left\{f_{+}(2 K \beta)\right\}-\sqrt{\frac{K}{2}} \ln \left\{f_{+}(\beta)\right\}, \\
& \Delta s=-\frac{1}{2} \ln \left\{f_{+}(2 K \beta)\right\}+\sqrt{\frac{K}{2}} \ln \left\{f_{+}(\beta)\right\} ;
\end{aligned}
$$

and the 2-shock curve $S_{2}$ based at $\left(r_{L}, s_{L}\right)$ is given for $0 \leqq \beta<\infty$, by

$$
\begin{aligned}
& \Delta r=-\frac{1}{2} \ln \left\{f_{+}(2 K \beta)\right\}-\sqrt{\frac{K}{2}} \ln \left\{f_{-}(\beta)\right\}, \\
& \Delta s=-\frac{1}{2} \ln \left\{f_{+}(2 K \beta)\right\}+\sqrt{\frac{K}{2}} \ln \left\{f_{-}(\beta)\right\} .
\end{aligned}
$$

Proof. For convenience, define

$$
w \equiv \frac{c-v}{c+v}
$$

so that

$$
\frac{v}{c}=\frac{1-w}{1+w}
$$

and by (72) and (73),

$$
r+s=\ln (w)
$$


Then by the definition of $\beta$ in (54) we have

$$
\begin{aligned}
K \beta\left(v, v_{L}\right) & =\frac{\left(\frac{v}{c}-\frac{v_{L}}{c}\right)^{2}}{\left(1-\frac{v^{2}}{c^{2}}\right)\left(1-\frac{v_{L}^{2}}{c^{2}}\right)} \\
& =\left(\frac{1-w}{1+w}-\frac{1-w_{L}}{1+w_{L}}\right)^{2} \frac{\left(1+w_{L}\right)^{2}}{4 w} \frac{\left(1+w_{L}\right)^{2}}{4 w_{L}} \\
& =\frac{1}{4}\left\{\sqrt{\frac{w}{w_{L}}}-\sqrt{\frac{w_{L}}{w}}\right\}^{2},
\end{aligned}
$$

which we can rewrite as

$$
\begin{aligned}
K \beta & =\frac{1}{4}\left\{\exp \left\{\frac{r-r_{L}}{2}+\frac{s-s_{L}}{2}\right\}-\exp \left\{-\frac{r-r_{L}}{2}-\frac{s-s_{L}}{2}\right\}\right\}^{2} \\
& =\sinh ^{2}\left(\frac{\Delta r}{2}+\frac{\Delta s}{2}\right) .
\end{aligned}
$$

Now solving for $w / w_{L}$ in (79) gives

$$
\frac{w}{w_{L}}=1+2 K \beta\left(1 \mp \sqrt{1+\frac{2}{K \beta}}\right) \equiv f_{\mp}(2 K \beta) \text {. }
$$

Note that $w$ is a monotone decreasing function of $v$, and $v$ decreases along $\mathbf{S}_{\mathbf{i}}$, $i=1,2$. Thus $w / w_{l} \geqq 1$ holds along $\mathbf{S}_{\mathbf{i}}$, so that we must choose the plus sign in (81) on both $i$-shock curves. On the other hand, by (53), along the shock curves we have

$$
\frac{\rho}{\rho_{L}}=f_{\mp}(\beta)
$$

where we take the plus sign when $i=1$ and the minus sign when $i=2$. Therefore, substituting (81) and (82) into (72) and (73), and choosing the appropriate plus and minus signs, gives (74)-(77). This completes the proof of Lemma 3.

What is interesting about (74)-(77) is that the differences $\Delta r$ and $\Delta s$ along a shock curve depend only on the parameter $\beta$, and thus the geometric shape of the shock curves in the $r s$-plane is independent of the base point $\left(r_{L}, s_{L}\right)$. This immediately implies that an $i$-shock curve based at one point in the rs-plane can be mapped by a rigid translation onto the $i$-shock curve based at any other point.

Lemma 4. The 2-shock curve based at an arbitrary point $\left(r_{L}, s_{L}\right)$ is the reflection in the rs-plane of the 1-shock curve based at the same point, where the axis of reflection is the line passing through $\left(r_{L}, s_{L}\right)$, parallel to the line $r=s$.

Proof. This follows immediately from (74)-(77) because using (62) we have

$$
f_{-}(\beta) f_{+}(\beta)=\left(1+\beta-\beta \sqrt{1+\frac{2}{\beta}}\right)\left(1+\beta+\beta \sqrt{1+\frac{2}{\beta}}\right)=1 .
$$

The following lemma gives further important geometric properties of the shock curves which we shall need. 
Lemma 5. The shock curves $S_{i}$ given in (74)-(77) define convex curves in the rs-plane, and moreover,

$$
0 \leqq \frac{d s}{d r}<\frac{\sqrt{2 K}-1}{-\sqrt{2 K}-1}<1
$$

all along a 1-shock curve $S_{1}$, and

$$
0 \leqq \frac{d r}{d s}<\frac{\sqrt{2 K}-1}{-\sqrt{2 K}-1}<1
$$

all along a 2-shock curve $S_{2}$.

Proof. By symmetry, it suffices to do the case $i=1$. Differentiating (74) with respect to $\beta$ gives

$$
\sqrt{\frac{2}{K}} \frac{d \Delta r}{d \beta}=-\frac{1}{\sqrt{2 \beta+\beta^{2}}}-\frac{1}{\sqrt{2 \beta+2 K \beta^{2}}},
$$

and differentiating (75) with respect to $\beta$ gives

$$
\sqrt{\frac{2}{K}} \frac{d \Delta s}{d \beta}=\frac{1}{\sqrt{2 \beta+\beta^{2}}}-\frac{1}{\sqrt{2 \beta+2 K \beta^{2}}},
$$

so that

$$
\frac{d \Delta s}{d \Delta r}=\frac{d s}{d r}=-\frac{\sqrt{2 \beta+2 K \beta^{2}}-\sqrt{2 \beta+\beta^{2}}}{-\sqrt{2 \beta+2 K \beta^{2}}-\sqrt{2 \beta+\beta^{2}}}<1,
$$

because $2 K \leqq 1$. To verify the convexity of $\mathbf{S}_{\mathbf{1}}$, we differentiate with respect to $\beta$, and simplify to get

$$
\frac{d}{d \beta} \frac{d s}{d r}=\frac{2(1-2 K)}{\beta^{2}}\left\{\sqrt{\frac{2}{\beta}+2 K}+\sqrt{\frac{2}{\beta}+1}\right\}^{-2}\left\{\sqrt{\frac{2}{\beta}+2 K} \sqrt{\frac{2}{\beta}+1}\right\}^{-1} \geqq 0 .
$$

Now (84) follows from the convexity of the shock curves together with the inequality

$$
\lim _{\beta \rightarrow \infty} \frac{d s}{d r}=\frac{\sqrt{2 K}-1}{-\sqrt{2 K}-1} \leqq 1 .
$$

A graph of the shock curves $S_{i}$ in the $(r, s)$-plane is given in Fig. 2.

As a final comment in this section, we note that we can obtain a corresponding parameterization of the shock curves for system (16) by taking the limit $c \rightarrow \infty$. To see this, note that taking the limit $c \rightarrow \infty$ in (72) and (73) we obtain

and

$$
\bar{r} \equiv c r \rightarrow v-\sigma \ln (\rho),
$$

$$
\bar{s} \equiv c s \rightarrow v+\sigma \ln (\rho),
$$

the right-hand sides being the Riemann invariants for system (16). Moreover, under this limit, (53) remains unchanged, and by (54),

$$
\beta \rightarrow \frac{\left(v-v_{L}\right)^{2}}{2 \sigma^{2}} .
$$




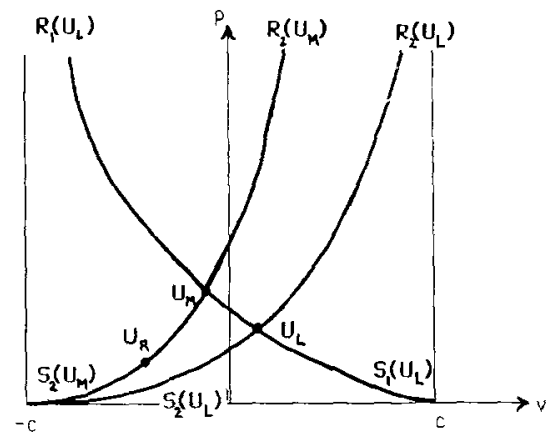

Fig. 3.

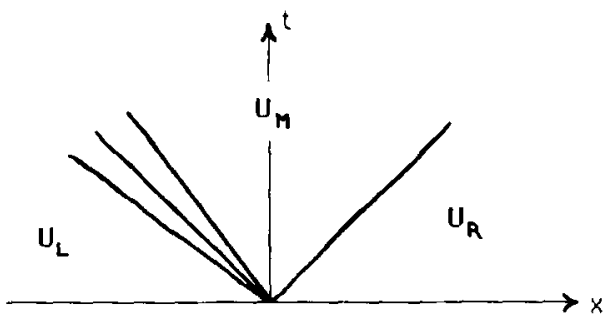

Fig. 4.

$U_{M} \in \mathbf{S}_{1}\left(U_{L}\right)$, and $U_{R} \in \mathbf{S}_{2}\left(U_{M}\right)$ then by (57),

$$
s_{1} \leqq \lambda_{1}\left(U_{M}\right)<\lambda_{2}\left(U_{M}\right) \leqq s_{2} .
$$

We state these results as a theorem (see Figs. 3 and 4):

Theorem 4. There exists a solution of the Riemann problem for system (14) in the case of an equation of state of the form $p=\sigma^{2} \rho, \sigma^{2}<c^{2}$, so long as $U_{L}$ and $U_{R}$ satisfy (88) and (89). Moreover, the solution is given by a 1-wave followed by a 2-wave, satisfies $\rho>0$, and all speeds are bounded by $c$. The solution is unique in the class of rarefaction waves and admissible shock waves.

We note that in the case of a polytropic equation of state, $\left(p(\rho)=\sigma^{2} \rho^{\gamma}, \gamma>1\right)$, the sound speed exceeds the speed of light for sufficiently large $\rho$, except in the limiting case $\gamma=1$. Thus, it is only in the limiting case $\gamma=1$ of a polytropic gas that a big data global existence theorem for the Riemann problem (such as Theorem 3 above) is possible without modifying the equation of state at large densities.

\section{The Initial Value Problem}

In this section we use the Glimm difference scheme to prove Theorem 1. We begin with a short discussion of this method.

Glimm's method is a procedure for obtaining solutions of the general initial value problem by constructing a convergent sequence of approximate solutions, the approximation scheme being based on the solution of the Riemann problem. 
to $U_{L}$, the existence and uniqueness of the solution of the Riemann problem for system (14) in the class of elementary waves follows by a general theorem of Lax which applies to any system of conservation laws which is strictly hyperbolic $\left(\lambda_{1}<\lambda_{2}\right)$ and genuinely nonlinear in each characteristic field. (See Theorem 3, and $[7,16]$.) We verify that for system (14), with $p=\sigma^{2} \rho$, the solution of the Riemann problem (87) can be (uniquely) constructed for all $U_{L}$ and $U_{R}$ provided that

$$
\rho_{L}>0, \quad \rho_{R}>0,
$$

and

$$
-c<v_{L}<c, \quad-c<v_{R}<c .
$$

To this end, fix $U_{L}$ and let $U \equiv U_{R}$ be variable. Let $\mathbf{R}_{\mathbf{i}} \equiv \mathbf{R}_{\mathbf{i}}\left(U_{L}\right)$ denote the $i$-rarefaction curve and $\mathbf{S}_{\mathbf{i}} \equiv \mathbf{S}_{\mathbf{i}}\left(U_{L}\right)$ the $i$-shock curve associated with the state $U_{L}$, [16]. The $i$-rarefaction curve $\mathbf{R}_{\mathbf{i}}$ at $U_{L}$ is defined to be the segment of the integral curve of the eigenvector $R_{i}$ which starts at $U_{L}$, and continues in the direction of increasing $\lambda_{i}$. Since the Riemann invariants $r$ and $s$, defined in (72) and (73), are constant on the 2- and 1-integral curves, respectively, it follows from genuine nonlinearity and Theorem 3 that

$$
\mathbf{R}_{1}\left(U_{L}\right)=\left\{U: s(U)=s\left(U_{L}\right) \text { and } r(U) \geqq r\left(U_{L}\right)\right\},
$$

and

$$
\mathbf{R}_{\mathbf{2}}\left(U_{L}\right)=\left\{U: r(U)=r\left(U_{L}\right) \text { and } s(U) \geqq s\left(U_{L}\right)\right\} .
$$

Note that for each state $U_{R} \in \mathbf{R}_{\mathbf{i}}\left(U_{L}\right)$, there is a rarefaction wave solution that solves the Riemann problem (87). Indeed, this is constructed by letting each state $U$ on $R_{i}$ between $U_{L}$ and $U_{R}$ propagate with speed $\lambda_{i}(U)$.

The $i$-shock curve $\mathbf{S}_{\mathrm{i}}$ is given in (74)-(77) of Lemma 3. If $U_{R} \in \mathbf{S}_{\mathbf{i}}\left(\mathbf{U}_{\mathbf{L}}\right)$, then the Riemann problem (87) is solved (in the weak sense) by a shock wave of speed $s_{i}$ (given by (63) and (64)), and the shock satisfies the Lax admissibility condition, (57). By Lax's theorem, the curve $S_{\mathrm{i}}$ makes $C^{2}$ tangency with the $i^{\text {th }}$ integral curve at $U_{L}$, and thus it follows from (72), (73) and (74)-77) that the composite wave curve $\mathbf{T}_{\mathbf{i}}$ defined by $\mathbf{T}_{\mathbf{i}} \equiv \mathbf{S}_{\mathbf{i}} \cup \mathbf{R}_{\mathbf{i}}$ is a $C^{2}$ curve for each $i=1,2$. Let $\mathbf{T}_{\mathbf{i}}(\varepsilon)$ denote the Euclidean arclength parameterization of the $i$-wave curve $\mathbf{T}_{\mathbf{i}}$, with $\varepsilon$ increasing with increasing $\lambda_{i}\left(\varepsilon>0\right.$ on $\mathbf{R}_{\mathbf{i}}, \varepsilon<0$ on $\mathbf{S}_{\mathbf{i}}$ ). It follows from (72), (73) and (74)-(77) that $\ln \rho$ increases monotonely from $-\infty$ to $+\infty$ along $\mathbf{T}_{\mathbf{1}}$, and decreases monotonely from $-\infty$ to $+\infty$ along $\mathbf{T}_{2}$; furthermore, $v$ increases monotonely from $-c$ to $+c$ along $\mathbf{T}_{1}$, and decreases monotonely from $+c$ to $-c$ along $\mathbf{T}_{2}$. The wave curves $\mathbf{T}_{\mathrm{i}}$ are sketched in Fig. 3.

To solve the Riemann problem, consider the wave curves $\mathbf{T}_{2}\left(U_{M}\right)$ for $U_{M} \in \mathbf{T}_{\mathbf{1}}\left(U_{L}\right)$. It is easily verified that any two such curves $\mathbf{T}_{\mathbf{2}}\left(U_{M}\right)$ and $\mathbf{T}_{\mathbf{2}}\left(U_{M}^{\prime}\right)$, $U_{M}, U_{M}^{\prime} \in \mathbf{T}_{1}\left(U_{L}\right)$, are nonintersecting, and that the set of all such curves covers the entire region $\rho>0,-c<v<c$ in the $\rho v$-plane in a 1-1 fashion. In particular we use the fact (see Lemma 5) that $|d s / d r|<1$ and $|d r / d s|<1$ all along the 1 -shock and 2-shock curves, respectively. Now for given states $U_{L}$ and $U_{R}$, let $U_{M} \in \mathbf{T}_{1}\left(U_{L}\right)$ denote the unique intermediate state such that $U_{R} \in \mathbf{T}_{2}\left(U_{M}\right)$. Then the unique solution of the Riemann problem in the class of elementary waves is given by a 1-wave connecting $U_{L}$ to $U_{M}$, followed by a 2-wave connecting $U_{M}$ to $U_{R}$. It remains only to verify that the 2 -wave speed is always greater than the 1-wave speed in this construction. But this follows directly from (57) of Lemma 2; i.e., if 


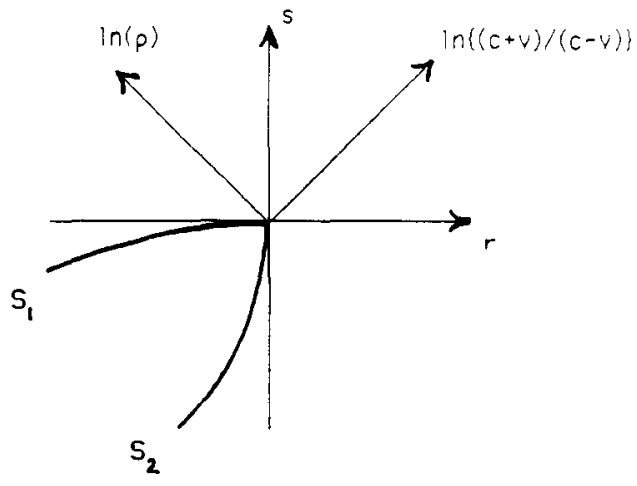

Fig. 2.

Thus multiplying by $c$ and taking the limit $c \rightarrow \infty$, the formulas (74)-(77) reduce to

$$
\begin{aligned}
& \Delta \bar{r}=-\frac{\sigma}{\sqrt{2}} \beta-\sigma \ln \left\{f_{+}(\beta)\right\}, \\
& \Delta \bar{s}=-\frac{\sigma}{\sqrt{2}} \beta+\sigma \ln \left\{f_{+}(\beta)\right\} ;
\end{aligned}
$$

and

$$
\begin{aligned}
& \Delta \bar{r}=-\frac{\sigma}{\sqrt{2}} \beta-\sigma \ln \left\{f_{-}(\beta)\right\}, \\
& \Delta \bar{s}=-\frac{\sigma}{\sqrt{2}} \beta+\sigma \ln \left\{f_{-}(\beta)\right\},
\end{aligned}
$$

respectively, and this gives parameterizations of the shock curves for system (16) that are equivalent to the formulas obtained by Nishida in [12].

\section{The Riemann Problem}

In this section we discuss the solution of the Riemann problem for system (14). In the next section we shall exploit special properties of the solution of the Riemann problem for this system to construct global weak solutions of the general initial value problem by means of the Glimm difference scheme, and to obtain the estimates (19), (20) and (21).

The Riemann problem is the initial value problem when the initial data $U_{0}(x) \equiv U\left(\rho_{0}(x), v_{0}(x)\right)$ consists of a pair of constant states $U_{L} \equiv U\left(\rho_{L}, v_{L}\right)$ and $U_{R} \equiv U\left(\rho_{R}, v_{R}\right)$ separated by a jump discontinuity at $x=0$,

$$
U_{0}(x)= \begin{cases}U_{L} & \text { if } x<0, \\ U_{R} & \text { if } x>0\end{cases}
$$

Note that, in view of Proposition 1, the conserved quantities $U_{L}$ and $U_{R}$ of system (13) are uniquely determined by $\left(\rho_{L}, v_{L}\right)$ and $\left(\rho_{R}, v_{R}\right)$. When $U_{R}$ is sufficiently close 
The scheme consists of approximating the solution at a fixed time level by piecewise constant states, so that one can solve the resulting Riemann problems thereby obtaining a sequence of elementary waves at that time level, the goal being to estimate the growth in the amplitude of these elementary waves as the waves interact during the time evolution of the solution. Glimm's method provides a scheme by which Riemann problems are re-posed at a subsequent time level according to a random choice of the state appearing in the waves of the previous time level. This has the advantage that waves at the subsequent time level are determined through the interaction of waves at the prior time level, and by this scheme, estimates on the amplitude changes in waves during interactions can then be used to estimate the growth of a solution in general. The natural measure of the amplitude, or strength of a wave $\gamma$, is the magnitude of the jump $\left|U_{R}-U_{L}\right| \equiv|\gamma|$. Thus, the total wave strength present in an approximate solution at time $t>0$ is given by

$$
\sum_{i}\left|\gamma_{i}\right|
$$

where the sum is over all waves present in the approximate solution at time $t$. The sum in (90) is equivalent to the total variation norm of the approximate solution at time $t>0$. The total variation of the waves will in general increase due to interactions because of the nonlinearity of the equations. Glimm showed that for a strictly hyperbolic, genuinely nonlinear (or linearly degenerate [16]) system, if the total initial strength of waves in an approximate solution is sufficiently small $\left(\sum_{i}\left|\gamma_{i}\right| \ll 1\right)$ at time $t=0$, then the total strength of waves at time $t>0$ is bounded by a constant times the initial strength. His method is to define a nonlocal functional $Q$, quadratic in wave strengths, which has the property that it decreases when waves interact, and moreover this decrease dominates the increase in total wave strength, when the initial wave strength is sufficiently small. This leads to the following theorem:

Theorem 5 (Glimm, [5]). Consider the initial value problem (23), (24) for a strictly hyperbolic, genuinely nonlinear system of conservation laws defined in a neighborhood of a state $U_{*}$. Then there exist constants $0<V \ll 1, C>0$, and a neighborhood $\mathbf{U}$ of $U_{*}$ such that, if the initial data $U_{0}$ lies in $\mathbf{U}$, and

$$
\operatorname{Var}\left\{U_{0}(\cdot)\right\}<V \text {, }
$$

then there exists a global weak solution $U(x, t)$ of (23), (24) obtained by Glimm's method, and this solution satisfies

$$
\operatorname{Var}\{U(\cdot, t)\} \leqq C \operatorname{Var}\left\{U_{0}(\cdot)\right\} .
$$

Glimm's method of analysis is the only method by which a time independent bound on wave strengths for a coupled nonlinear system of conservation laws has been rigorously proven. Glimm's method is also the only numerical method that has been proved to converge for the $3 \times 3$ classical Euler equations of gas dynamics. In this section we show that system (14) has the very special property that when $p=\sigma^{2} \rho$, the total variation of $\ln (\rho)$ is nonincreasing (in time) when elementary waves interact. Thus, $\operatorname{Var}\{\ln (\rho)\}$ plays the role of an energy function, and one can use this in place of the nonlocal functional $Q$ in Glimm's method. This fact enables us to prove Theorem 1, a large data existence theorem, in this special case. (This idea is due to Nishida [12].) 
We now define the Glimm difference scheme for system (14) in detail, and prove Theorem 1. Let $\Delta x$ denote a mesh length in $x$ and $\Delta t$ a mesh length in $t$, and let $x_{j} \equiv j \Delta x$ and $t_{n} \equiv n \Delta t$ denote the mesh points in an approximate solution. Let $U_{0}(x) \equiv U\left(\rho_{0}(x), v_{0}(x)\right)$ denote initial data for system (14) satisfying $\rho_{0}(x)>0$, $-c<v_{0}(x)<c$. To define the Glimm scheme approximate solution $U_{\Delta x}(x, t)$, we approximate the initial data by the piecewise constant states, $U_{i}^{0}=U_{0}\left(x_{j}+\right)$. To start the scheme, define

$$
U_{\Delta x}(x, 0)=U_{j}^{0}, \quad \text { for } x_{j} \leqq x<x_{j+1} .
$$

Now assume that the approximate solution $U_{\Delta x}$ has been defined for $t \leqq t_{n-1}$, and that the solution at time $t=t_{n-1}$ is given by piecewise constant states

$$
U_{\Delta x}\left(x, t_{n-1}\right)=U_{j}^{n-1}, \text { for } x_{j} \leqq x<x_{j+1} \text {. }
$$

In order to complete the definition of $U_{\Delta x}$ by induction, it suffices to define $U_{\Delta x}(x, t)$ for $t_{n-1}<t \leqq t_{n}$. For $t_{n-1}<t<t_{n}$, let $U_{\Delta x}(x, t)$ be obtained by solving the Riemann problems posed at time $t=t_{n-1}$ as in Theorem 3. Note that since all wave speeds are bounded by $c$, we assume that

$$
\Delta x / \Delta t \geqq 2 c,
$$

in order to insure that waves do not interact within one time step. Now to re-pose the constant states and the corresponding Riemann problems at time level $t_{n}$ in the approximate solution, let $\mathbf{a} \equiv\left\{a_{k}\right\} \in \mathbf{A}$ denote a (fixed) random sequence, $0<a_{k}<1$, where $\mathbf{A}$ denotes the infinite product of intervals $(0,1)$ endowed with Lebesgue measure, $1<k<\infty$. Then define

$$
U_{\Delta x}\left(x, t_{n}+\right)=U_{j}^{n}, \quad \text { for } x_{j} \leqq x<x_{j+1},
$$

where

$$
U_{j}^{n}=U_{\Delta x}\left(x_{j}+a_{n} \Delta x, t_{n}-\right) .
$$

This completes the definition of the approximate solution $U_{\Delta x}$ by induction. (Note that $U_{\Delta x}$ depends on the choice of $a \in \mathbf{A}$.) The restriction on the random sequence a will come at the end. The important point is that the waves in the solution $U_{\Delta x}$ at mesh point $\left(x_{j}, t_{n}\right)$ solve the Riemann problem with $U_{L}=U_{\Delta x}\left(x_{j-1}+a_{n} \Delta x, t_{n}-\right)$ and right state $U_{R}=U_{\Delta x}\left(x_{j}+a_{n} \Delta x, t_{n}-\right)$, these being states that appear in the waves of the previous time level. In the case of system (14), we modify the above definition of wave strength $|\gamma|$ by defining

$$
|\gamma|=\left|\ln \left(\rho_{R}\right)-\ln \left(\rho_{L}\right)\right|,
$$

where $U_{L}$ is the left state and $U_{R}$ the right state of the wave $\gamma$. The proof of Theorem 1 is based on the following lemma:

Lemma 6. Let $U_{L}, U_{M}$ and $U_{R}$ denote three arbitrary states satisfying (88) and (89). Let $\alpha_{i}, \beta_{i}$ and $\gamma_{i}$ denote the waves that solve the Riemann problems $\left[U_{L}, U_{M}\right]$, $\left[U_{M}, U_{R}\right]$ and $\left[U_{L}, U_{R}\right]$, respectively, $i=1,2$. Then

$$
\left|\gamma_{1}\right|+\left|\gamma_{2}\right| \leqq\left|\alpha_{1}\right|+\left|\alpha_{2}\right|+\left|\beta_{1}\right|+\left|\beta_{2}\right|
$$

Proof. Lemma 6 follows from the special geometry of the shock curves that was obtained in Sect. 5. The important point is that the graphs of the shock curves 
$\mathbf{S}_{i}\left(U_{L}\right)$ in $r s$-plane (the plane of Riemann invariants (72) and (73)) have the same geometric shape, independent of $U_{L}$; and the 1-shock curves are reflections of the 2-shock curves about the line $r=s$. It is also important that Lemma 5 holds: namely, $0 \leqq|d s / d r|<1$ all along the 1 -shock curves $\mathbf{S}_{\mathbf{1}}$, and $0 \leqq|d r / d s|<1$ along $\mathbf{S}_{2}$. In particular, these latter conditions imply that the interaction of shock waves of the same family produces a stronger shock in the same family, together with a rarefaction wave of the opposite family. This special geometry is enough to imply that the sum of the strengths of the waves as projected onto the $(s-r)$-axis (recall that $s-r=\sqrt{\frac{K}{2}} \ln (\rho)$ ) is the same in the outgoing waves as in the incoming waves for any interaction $\left[U_{L}, U_{M}\right],\left[U_{M}, U_{R}\right] \rightarrow\left[U_{L}, U_{R}\right]$ - except in the case when waves of the same family cancel, in which case the sum decreases. Note that Lemma 5 guarantees that the strength $|\gamma|$ is monotone in $U_{R}$ along $S_{i}$, $i=1,2$, and this is needed to ensure that the Euclidean strength $\left|U_{L}-U_{R}\right|$ across a wave $\gamma$ can be bounded by the strength of the wave as defined in (93). In Fig. 5, estimate (94) is demonstrated in several examples of wave interactions. The general case is a straightforward generalization of these, and we omit the details. $[12,16]$.

Now let $\gamma_{j i}^{n}$ denote the $i$-wave that appears in the solution of the Riemann problem posed at the mesh point $\left(x_{j}, t_{n}\right)$ in the approximate solution $U_{\Delta x}, i=1,2$.
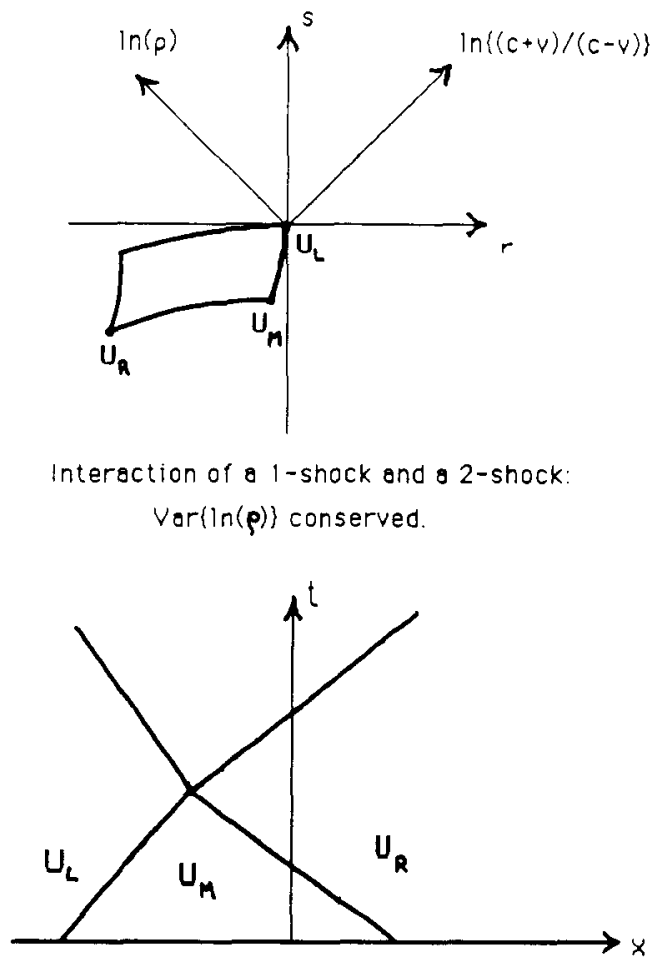

Fig. 5a. 


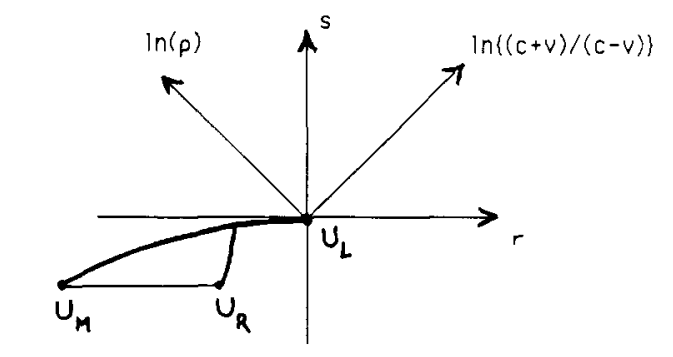

Interaction of a 1 -shock and a 1 -rarefaction wave:
\[ \operatorname{var}\{\ln (p)\} \text { decreases. } \]

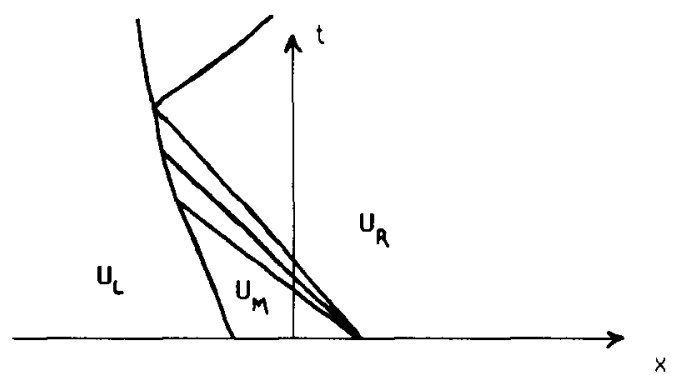

Fig. 5b.

Lemma 7. Let $U_{A x}$ denote an approximate solution of the random choice method for initial data satisfying (88) and (89). Then

$$
\sum_{-\infty<j<\infty, i=1,2}\left|\gamma_{j i}^{n+1}\right| \leqq \sum_{-\infty<j<\infty, i=1,2}\left|\gamma_{j i}^{n}\right| .
$$

Proof. Consider the portions of the elementary waves $\gamma_{j-1, i}^{n}, \gamma_{j i}^{n}$ and $\gamma_{j+1, i}^{n}$ that, at time level $t=t_{n+1}$, lie within the spacial interval $\left[x_{j-1}+a_{n+1} \Delta x, x_{j}+a_{n+1} \Delta x\right)$. Let these waves be denoted $\alpha_{j-1, i}^{n}, \bar{\gamma}_{j, i}^{n}$ and $\beta_{j+1, i}^{n}$ according to whether the wave emanates from the mesh point $\left(x_{j-1}, t_{n}\right),\left(x_{j}, t_{n}\right)$ or $\left(x_{j+1}, t_{n}\right)$, respectively. Then the waves $\alpha_{j-1, i}^{n}, i=1,2$, are either all zero strength waves, (the random point lies closer to $x=x_{j}$ than the states in the waves $\alpha_{j-1, i}^{n}$ at time level $\left.t=t_{n+1}\right)$ ), or else they are the waves that solve the Riemann problem with left state $U_{L} \equiv U_{\Delta x}\left(x_{j-1}+a_{n+1} \Delta x, t_{n+1}-\right)$, and right state $U_{j-1}^{n}$; the waves $\beta_{j+1, i}^{n}$ are similarly all zero or else they solve the Riemann problem with left state $U_{j}^{n}$ and right state $U_{R} \equiv U_{\Delta x}\left(x_{j}+a_{n+1} \Delta x, t_{n+1}-\right)$; and $\bar{\gamma}_{j i}^{n}$ solve the Riemann problem with left state either $U_{L}$ or $U_{j-1}^{n}$ and right state either $U_{j}^{n}$ or $U_{L}$, depending on where the random points $\left(x_{j-1}+a_{n+1} \Delta x, t_{n+1}-\right)$ and $\left(x_{j}+a_{n+1} \Delta x, t_{n+1}-\right)$ fall relative to the waves $\gamma_{j i}^{n}$ at time level $t_{n+1}$. Note that in this notation, the waves $\gamma_{j i}^{n+1}$ solve the Riemann problem $\left[U_{L}, U_{R}\right]$. Thus by Lemma 6 we can conclude that

$$
\sum_{i=1,2}\left|\gamma_{j i}^{n+1}\right| \leqq \sum_{i=1,2}\left\{\left|\alpha_{j-1, i}^{n}\right|+\left|\bar{\gamma}_{j i}^{n}\right|+\left|\beta_{j+1, i}^{n}\right|\right\}
$$


But summing $j$ from $-\infty$ to $+\infty$ in (96) and rearranging terms gives

$$
\sum_{i, j}\left|\gamma_{j i}^{n+1}\right| \leqq \sum_{i, j}\left\{\left|\alpha_{j, i}^{n}\right|+\left|\bar{\gamma}_{j i}^{n}\right|+\left|\beta_{j, i}^{n}\right|\right\} \leqq \sum_{i, j}\left|\gamma_{j i}^{n}\right|
$$

the latter inequality holding because, by construction,

$$
\left|\alpha_{j i}^{n}\right|+\left|\bar{\gamma}_{j i}^{n}\right|+\left|\beta_{j i}^{n}\right|=\left|\gamma_{j i}^{n}\right| \text {. }
$$

This completes the proof of Lemma 6.

We now complete the proof of Theorem 1. First, since $\ln (\rho)$ is monotone along the wave curves $\mathbf{T}_{\mathbf{i}}$, it follows that at time level $t \in\left(t_{n}, t_{n+1}\right)$ in an approximate solution $U_{A x}$, the sum of the strengths of the waves at time $t$ is equivalent to the total variation in $\ln (\rho)$ of the approximate solution at time $t$ :

$$
\operatorname{Var}\left\{\ln \left(\rho_{\Delta x}(\cdot, t)\right)\right\}=\sum_{i, j}\left|\gamma_{j i}^{n}\right|
$$

Thus, in the approximate solution,

$$
\operatorname{Var}\left\{\ln \left(\rho_{\Delta x}(\cdot, t+)\right)\right\} \leqq \operatorname{Var}\left\{\ln \left(\rho_{\Delta x}(\cdot, s+)\right)\right\},
$$

whenever $s \leqq t$. We now note that by Helly's theorem [16], $L^{1}$ limits of functions of uniformly bounded variation satisfy the same variation bound. This gives the first inequality (19) of Theorem 1 for any weak solution $U(x, t)=U(\rho(x, t), v(x, t))$ obtained as an $L^{1}$ limit of approximate solutions $U_{\Delta x}$ as $\Delta x \rightarrow 0$. Thus we show that (19) of Theorem 1 is a consequence of the following lemma:

Lemma 8 (Glimm, [5]). Assume that the approximate solution $U_{\Delta x}$ satisfies

$$
\operatorname{Var}\left\{U_{\Delta x}(\cdot, t)\right\}<V<\infty
$$

for all $t \geqq 0$. Then there exists a subsequence of mesh lengths $\Delta x \rightarrow 0$ such that $U_{\Delta x} \rightarrow U$, where $U(x, t)$ also satisfies (99). The approximate solutions converge pointwise a.e., and in $L_{\mathrm{loc}}^{1}$ at each time, uniformly on bounded $x$ and $t$ sets. Moreover, there exists a set $\mathbf{N} \subset \mathbf{A}$ of Lebesgue measure zero such that, if $\mathbf{a} \in \mathbf{A}-\mathbf{N}$, then $U(x, t)$ is a weak solution of the initial value problem (14), (15).

Using Lemma 8, the proof of (19) and (20) of Theorem 1 is completed once we show that the estimates (17) and (18) imply (99) for the approximate Glimm scheme solutions. For this, note that (17) and (18) imply that there exist states $\rho_{\infty}=\lim _{x \rightarrow \infty} \rho_{0}(x)$ and $u_{\infty}=\lim _{x \rightarrow \infty} u_{0}(x)$. But (18) implies that the total variation in $\ln \left\{\frac{c+v}{c-v}\right\}$ is finite at time $t=0+$ in the approximate solution $U_{\Delta x}$, and thus

$$
\operatorname{Var}\left\{\ln \left(\rho_{\Delta x}(\cdot, 0+)\right)\right\}<V_{0},
$$

where $V_{0}$ depends only on the initial total variation bounds in (17) and (18). Thus, by (98),

$$
\operatorname{Var}\left\{\ln \left(\rho_{\Delta x}(\cdot, t+)\right)\right\}<V_{0},
$$

for all positive times $t>0$. But it follows directly from (85) and (84) of Lemma 5 that the variation in $\ln \left\{\frac{c+v}{c-v}\right\}$ is bounded uniformly by the variation in $\ln (\rho)$ across every elementary wave in each approximate solution $U_{\Delta x}$. Thus (100) 
implies that

$$
\operatorname{Var}\left\{\ln \left(\frac{c+v}{c-v}\right)(\cdot, t+)\right\}<V_{1},
$$

at each $t>0$ in an approximate solution $U_{\Delta x}$, where $V_{1}$ depends only on $V_{0}$. It now follows from (100) and (101) that for every $\Delta x$ and $\mathbf{a} \in \mathbf{A}, \rho_{\infty}=\lim _{x \rightarrow \infty} \rho_{\Delta x}(x, t)$ and $v_{\infty}=\lim _{x \rightarrow \infty} v_{\Delta x}(x, t)$. This means that $\left(\rho_{\infty}, v_{\infty}\right)$ is a constant state appearing in the approximate solutions at each fixed time level. This together with (100) and (101) implies that there exists a constant $M>0$ such that

$$
1 / M<\rho_{\Delta x}(x, t)<M
$$

and

$$
-c+1 / M<v_{\Delta x}(x, t)<c-1 / M
$$

for all $x$ and $t \geqq 0$, uniformly in $\Delta x$. The desired result (99) follows from these latter two bounds because, in light of Proposition 1, the Jacobian determinant $\left|\frac{\partial\left(U_{1}, U_{2}\right)}{\partial(\rho, v)}\right|$ is bounded away from zero (uniformly in $\left.\Delta x\right)$ on the image of the approximate solutions $U_{A x}$. Thus by Lemma 8 there exists a subsequence of mesh lengths $\Delta x \rightarrow 0$ such that $U_{\Delta x} \rightarrow U(\rho(x, t), v(x, t))$ where $U$ satisfies (99), (19) and (20), and the convergence is pointwise a.e., and in $L_{\text {loc }}^{1}$ at each time, uniformly on bounded $x$ and $t$ sets. By Proposition 2, $V_{0}$ and $V_{1}$ must be Lorentz invariant constants.

\section{Appendix I}

In this section we show that the transformation properties of characteristics and shocks in a relativistic system of conservation laws follows directly from the covariance properties of the Rankine-Hugoniot jump relations alone. In particular, we show that the characteristic curves and shock curves associated with a system of conservation laws $\operatorname{div} T=0$, transform, under general nonlinear spacetime coordinate transformations, like the level curve of a scalar function. We assume that the divergence is taken with respect to a Lorentz metric $g$ defined in four dimensional spacetime. As a consequence, we show that the wave speeds $\lambda_{i}$ and the shock speeds $s_{i}$, defined for systems (9) and (14), transform according to the special relativistic velocity transformation law (12).

Thus, if $g_{i j}$ denotes a fixed Lorentzian metric defined on a four dimensional spacetime $\mathbf{x} \equiv\left(x^{0}, \ldots, x^{3}\right), x^{0}=c t$, let $\Gamma_{j k}^{i}$ denote the Christoffel symbols which define the unique symmetric connection associated with $g_{i j}$, namely

$$
\Gamma_{j k}^{i} \equiv \frac{1}{2} g^{i \sigma}\left\{g_{\sigma j, k}+g_{k \sigma, j}-g_{j k, \sigma}\right\} \text {. }
$$

Let $T_{i j}$ be a symmetric $(0,2)$-tensor which we take to be the stress energy tensor for some field in spacetime. Conservation of energy-momentum based on the metric $g$ then reads div $T=0$, where the covariant divergence is given in coordinates by $[20,4]$

$$
\operatorname{div} T \equiv T_{j ; \sigma}^{\sigma} \equiv T_{j, \sigma}^{\sigma}+\Gamma_{\tau \sigma}^{\sigma} T_{j}^{\tau}-\Gamma_{j \sigma}^{\tau} T_{\tau}^{\sigma}
$$

Here, ", $i$ " denotes $\partial / \partial x^{i}$ and "; $i$ " denotes the covariant derivative. 
Definition 1. Assume that $T_{i j}$ (defined in a given coordinate system $\mathbf{x}$ ) is smooth except for a jump discontinuity across a smooth surface $\phi(\mathbf{x})=0$, where $d \phi \equiv n_{\sigma} d x^{\sigma} \neq 0$. Then we say that $T$ is a weak solution of $\operatorname{div} T=0$ if this equation holds away from the surface $\phi(\mathbf{x})=0$, and across the surface the following (Rankine-Hugoniot) jump conditions hold:

$$
\left[T_{j}^{\sigma} n_{\sigma}\right]=0
$$

Here, as usual, the square brackets around a quantity denote the jump in the quantity across the surface $\phi=0$,

$$
\left[T_{j}^{\sigma} n_{\sigma}\right] \equiv\left(T_{j}^{\sigma} n_{\sigma}\right)_{R}-\left(T_{j}^{\sigma} n_{\sigma}\right)_{L}=\left[T_{j}^{\sigma}\right] n_{\sigma} .
$$

One can show that the jump relation is implied by the weak formulation of $\operatorname{div} T=0$ in the sense of the theory of distributions [16], and implies conservation of the physical energy-momentum across the surface of discontinuity $\phi=0$. The equivalence of the weak formulation follows from integration by parts, observing that the non-divergence terms $\Gamma_{\tau \sigma}^{\sigma} T_{j}^{\tau}-\Gamma_{j \sigma}^{\tau} T_{t}^{\sigma}$ contain no derivative of $T$.

From the jump conditions we obtain the following proposition:

Proposition A1. A shock surface transforms (under arbitrary nonlinear changes of spacetime coordinates) as the level curve of a scalar function defined on spacetime.

Proof. If $\left[T_{j}^{i}\right] n_{i}=0$ holds on the surface $\phi=0$ in one coordinate system $x$ where $d \phi=n_{i} d x^{i}$, then it holds in every other coordinate system because $T$ transforms like a $(1,1)$-tensor, and $d \phi$ is a 1 -form.

Now restricting to a 2-dimensional Minkowski spacetime, so that $g_{i k} \equiv \eta_{i j}$, Proposition A1 implies that the shock curve has tangent vector $d \mathbf{x} / d \tau=X$ if and only if $n_{i} d x^{i}(X)=0$, where $n_{i} d x^{i}=d \phi$ and $\phi$ is a function constant along the shock curve. Thus, letting $X^{i}$ denote the components of $X$, we conclude that the shock speed $s=d x^{1} / d t$ is given by

$$
s=c \frac{X^{1}}{X^{0}}=-c \frac{n_{0}}{n_{1}} .
$$

Proposition A2. Under Lorentz transformations, the shock speeds s transform according to the velocity transformation rule (12).

Proof. Consider a Lorentz transformation taking the unbarred coordinates $x^{i}$ to the barred coordinates $\bar{x}^{\alpha}$, such that the barred frame moves with velocity $\mu$ as measured in the unbarred frame $x^{i}$. Then

$$
\bar{x}^{\alpha}=\Lambda(\mu)_{i}^{\alpha} x^{i},
$$

where

$$
\Lambda(\mu)_{i}^{\alpha}=\left[\begin{array}{cc}
\cosh (\theta) & \sinh (\theta) \\
\sinh (\theta) & \cosh (\theta)
\end{array}\right],
$$

and $\tanh (\theta)=\mu / c,[20]$. Then the vector $X$, tangent to the shock curve, transforms as

$$
\bar{X}^{\alpha}=\Lambda(\mu)_{i}^{\alpha} X^{i}
$$


Thus in the barred coordinates, the shock speed is given by

$$
\bar{s}=c \frac{\bar{X}^{1}}{X^{0}}=c \frac{X^{0} \cosh (\theta)+X^{1} \sinh (\theta)}{X^{0} \sinh (\theta)+X^{1} \cosh (\theta)}=\frac{\mu+s}{1+\frac{\mu \nu}{c^{2}}} .
$$

This completes the proof of Proposition A2.

Proposition A3. The characteristic curves for a system of conservation laws (23) transform as level curves of functions in physical space. Moreover, under Lorentz transformations, the wave speeds $\lambda_{i}$ (the eigenvalues of $d F$ ) transform according to the velocity transformation law (12).

Proof. By Theorem 2, $\lambda_{i}=\lim _{\varepsilon \rightarrow 0} s_{i}(\varepsilon)$. Thus, by continuity, $\lambda_{i}$ transforms as a velocity (12) under Lorentz transformations because $s_{i}(\varepsilon)$ does for each fixed $\varepsilon$. Since the characteristic curves are given by $d x^{1} / d t=\lambda_{i}$ and $\lambda_{i}$ transforms as a velocity, it follows that the characteristic curves must transform like level curves of functions. Thus by duality, in two dimensional spacetime, the tangents to the shock curves and characteristic curves transform like vectors.

\section{References}

1. Anile, A.M.: Relativistic Fluids and Magneto-Fluids. Cambridge Monographs on Mathematical Physics, Cambridge: Cambridge University Press 1989

2. Christodoulou, D.: Private communication

3. Courant, R., Friedrichs, K.O.: Supersonic Flow and Shock Waves. New York: Wiley 1948

4. Dubrovin, B.A., Fomenko, A.T., Novikov, S.P.: Modern Geometry-Methods and Applications. Berlin, Heidelberg, New York: Springer 1984

5. Glimm, J.: Solutions in the large for nonlinear hyperbolic systems of equations. Commun. Pure Appl. Math. 18, 697-715 (1965)

6. Johnson, M., McKee, C.: Relativistic hydrodynamics in one dimension. Phys. Rev. D, 3, no. 4, 858-863 (1971)

7. Lax, P.D.: Hyperbolic systems of conservation laws, II. Commun. Pure Appl. Math. 10, 537-566 (1957)

8. Liu, T.P.: Private communication

9. Luskin, M., Temple, B.: The existence of a global weak solution of the waterhammer problem. Commun. Pure Appl. Math. 35, 697-735 (1982)

10. McVittie, G.C.: Gravitational collapse to a small volume. Astro. Phys. J. 140, 401-416 (1964)

11. Misner, C., Sharp, D.: Relativistic equations for adiabatic, spherically symmetric gravitational collapse. Phys. Rev. 26, 571-576 (1964)

12. Nishida, T.: Global solution for an initial boundary value problem of a quasilinear hyperbolic system. Proc. Jap. Acad. 44, 642-646 (1968)

13. Nishida, T., Smoller, J.: Solutions in the large for some nonlinear hyperbolic conservation laws. Commun. Pure Appl. Math. 26, 183-200 (1973)

14. Oppenheimer, J.R., Snyder, J.R.: On continued gravitational contraction. Phys. Rev. 56, 455-459 (1939)

15. Oppenheimer, J.R., Volkoff, G.M.: On massive neutron cores. Phys. Rev. 55, 374-381 (1939)

16. Smoller, J.: Shock Waves and Reaction Diffusion Equations. Berlin, Heidelberg, New York: Springer 1983

17. Taub, A.: Approximate solutions of the Einstein equations for isentropic motions of planesymmetric distributions of perfect fluids. Phys. Rev. 107, no. 3, 884-900 (1957) 
18. Thompson, K.: The special relativistic shock tube. J. Fluid Mech. 171, 365-375 (1986)

19. Tolman, R.: Relativity, Thermodynamics and Cosmology. Oxford: Oxford University Press 1934

20. Weinberg, S.: Gravitation and Cosmology: Principles and Applications of the General Theory of Relativity. New York: Wiley 1972

Communicated by S.-T. Yau 